\title{
Mitochondrial Aurora kinase A induces mitophagy by interacting with MAP1LC3 and Prohibitin 2
}

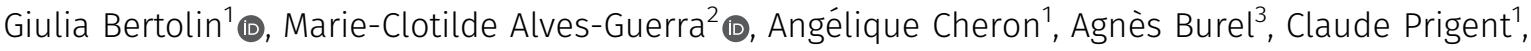 \\ Roland Le Borgne $\mathbb{1}^{1}$, Marc Tramier $^{1}$ (])
}

\begin{abstract}
Epithelial and haematologic tumours often show the overexpression of the serine/threonine kinase AURKA. Recently, AURKA was shown to localise at mitochondria, where it regulates mitochondrial dynamics and ATP production. Here we define the molecular mechanisms of AURKA in regulating mitochondrial turnover by mitophagy. AURKA triggers the degradation of Inner Mitochondrial Membrane/ matrix proteins by interacting with core components of the autophagy pathway. On the inner mitochondrial membrane, the kinase forms a tripartite complex with MAP1LC3 and the mitophagy receptor PHB2, which triggers mitophagy in a PARK2/Parkin-independent manner. The formation of the tripartite complex is induced by the phosphorylation of PHB2 on Ser39, which is required for MAP1LC3 to interact with PHB2. Last, treatment with the PHB2 ligand xanthohumol blocks AURKA-induced mitophagy by destabilising the tripartite complex and restores normal ATP production levels. Altogether, these data provide evidence for a role of AURKA in promoting mitophagy through the interaction with PHB2 and MAP1LC3. This work paves the way to the use of function-specific pharmacological inhibitors to counteract the effects of the overexpression of AURKA in cancer.
\end{abstract}

DOI 10.26508/lsa.202000806 | Received 5 June 2020 | Revised 10 February 2021 | Accepted 25 March 2021 | Published online 5 April 2021

\section{Introduction}

AURKA is a serine/threonine kinase with multiple functions during interphase and cell division. It is frequently overexpressed in solid tumours and during haematological malignancies, where it correlates with poor patient survival and resistance to treatments (Farag, 2011; Nikonova et al, 2013; Bertolin \& Tramier, 2020). AURKA was originally found at centrosomes and at the mitotic spindle in Xenopus laevis (Paris \& Philippe, 1990; Glover et al, 1995; Andrésson \& Ruderman, 1998; Roghi et al, 1998), and in Drosophila melanogaster (Giet et al, 2002; Lee et al, 2006; Wang et al, 2006; Wirtz-Peitz et al, 2008). AURKA was then reported to localise to other subcellular locations such as the nucleus (Zheng et al, 2016), primary cilia (Pugacheva \& Golemis, 2005; Pugacheva et al, 2007; Kinzel et al, 2010; Lee et al, 2012; Mergen et al, 2013), and more recently at mitochondria (Bertolin et al, 2018; Grant et al, 2018). At this compartment, we and others reported that AURKA regulates two key mitochondrial functions as organelle dynamics and ATP production throughout the cell cycle (Kashatus et al, 2011; Bertolin et al, 2018; Grant et al, 2018). Given these multiple locations, our knowledge of the subcellular interactome of AURKA and the new intracellular functions for this kinase is constantly growing (Bertolin \& Tramier, 2020).

Mitochondria are dynamic organelles continuously undergoing cycles of fusion and fission to meet the energy requirements of the cell (Mishra \& Chan, 2016). Concomitantly to these functions, turnover programs are also activated to selectively eliminate defective portions of the mitochondrial network. These programs mainly rely on mitochondrial-specific autophagy (mitophagy), and they are necessary for cell homeostasis in both physiological and pathological paradigms (Pickrell \& Youle, 2015). The mitophagy pathway has been extensively characterised in paradigms of Parkinson's disease. The Parkinson's disease-linked proteins PARK2-Parkin and PTEN-induced kinase 1 (PINK1) directly interact with various components of the autophagy machinery, and constitute a common pathway triggering mitochondrial turnover (Youle \& Narendra, 2011). Downstream of PARK2-Parkin and PINK1, the molecular actors regulating organelle-specific elimination pathways like mitophagy are key components of the autophagy pathway. Upon the induction of mitophagy, a family of small ubiquitin-like modifiers-MAP1LC3 and GABARAP proteins-are modified through the addition of a phosphatidylethanolamine molecule (Nakatogawa, 2013). This modification hallmarks for the activation of MAP1LC3 on the nascent autophagosomal membrane (Mercer et al, 2018). Together with MAP1LC3 lipidation, the serine/threonine kinase UNC-51-like kinase 1 (ULK1) and ATG9 proteins independently contribute to the early steps of autophagosome formation during mitophagy (Hurley \& Young, 2017). Organelle-specific autophagy then uses selective downstream adaptors and effectors such as Sequestosome-1 (SQSTN1/p62) (Geisler et al, 2010), neighbour of BRCA1 gene 1 (NBR1) (Isakson et al, 2013), nuclear dot-protein 52 ${ }^{1}$ University of Rennes, Centre National de la Recherche Scientifique (CNRS), (IGDR) Genetics and Development Institute of Rennes, Unité Mixte de Recherche (UMR) 6290,
Rennes, France ${ }^{2}$ Université de Paris, Institut Cochin, Institut National de la Santé et de la Recherche Médicale (INSERM), CNRS, Paris, France ${ }^{3}$ University of Rennes, MRic
CNRS, INSERM, Structure Fédérative de Recherche (SFR) Biosit, UMS 3480, Rennes, France

Correspondence: giulia.bertolin@univ-rennes1.fr 
(NDP52), and Optineurin (OPTN) (Lazarou et al, 2015), which are recruited to defective mitochondria and are necessary for their consequent elimination within autophagosomes.

Although less extensively characterised, physiological paradigms for mitophagy exist in specific cell types. For instance, the Outer Mitochondrial Membrane (OMM) protein Nip3-like protein $X$ (NIX) interacts with MAP1LC3 and its homologue GABARAP to trigger the elimination of mitochondria in red blood cells (Kim et al, 2007; Schwarten et al, 2009; Zhang et al, 2009; Mortensen et al, 2010; Novak et al, 2010). More recently, Prohibitin 2 (PHB2) was identified as the receptor of MAP1LC3 on the inner mitochondrial membrane (IMM) (Wei et al, 2017). In the same study using Caenorhabditis elegans, PHB2 was shown to be essential both in PARK2-Parkin/PINK1dependent mitophagy in cultured cells and for the clearance of paternal organelles upon fertilisation, a physiological paradigm of mitochondrial turnover.

We here discover that overexpressed AURKA triggers mitophagy by forming a tripartite complex with MAP1LC3 and PHB2 at mitochondria. AURKA induces organelle turnover independently of PARK2/Parkin. Last, we identify a the PHB2 ligand xanthohumol as a potential pharmacological strategy to block mitophagy by destabilising the tripartite complex in cells overexpressing AURKA. Whereas the overexpression of AURKA exacerbates mitochondrial ATP production (Bertolin et al, 2018), xanthohumol restores normal ATP levels in cells, further corroborating the link between mitophagy and mitochondrial energy production.

\section{Results}

\section{Overexpressed AURKA triggers the recruitment of MAP1LC3 to mitochondria and it induces mitochondrial mass loss}

In epithelial cancers, AURKA is commonly overexpressed. It is known that unbalancing AURKA protein abundance towards overexpression leads to intracellular abnormalities as defective mitotic spindles, chromosomal misattachments and supernumerary centrosomes. Yet, the consequences of AURKA overexpression on mitochondria as a potential cancer-promoting event are still fairly unknown. We previously reported that AURKA elongates the mitochondrial network when overexpressed (Bertolin et al, 2018). Under the same experimental conditions, we detected a partial loss of mitochondrial mass in MCF7 cells-an epithelial breast cancer cell line (Fig 1A), when measuring the area covered by the mitochondrial matrix marker PMPCB (Bertolin et al, 2013). To evaluate whether PMPCB loss is directly linked to the localisation of AURKA at mitochondria, we overexpressed the cytosolic-only AURKA variant $\triangle$ Nter. As previously reported, this construct lacks the mitochondrial-targeting sequence of AURKA (Bertolin et al, 2018). Conversely to the normal protein, AURKA $\triangle$ Nter did not induce any loss of mitochondrial mass (Figs $1 A$ and S1A). No mitochondrial mass loss was observed when endogenous AURKA was depleted by siRNA (Fig 1A), indicating that endogenous levels of AURKA in MCF7 cells are too low to induce a mitochondrial mass loss. On the contrary, siRNA-mediated down-regulation of AURKA in triple-negative T47D breast carcinoma cells, which physiologically express high levels of AURKA (Bertolin et al, 2018), show a partial increase in mitochondrial mass compared with control cells (Fig S1B). This reinforces the observation that the effect of AURKA on mitochondrial mass depends on the relative abundance of the kinase. To further validate the loss of mitochondrial mass observed in cells overexpressing AURKA, we used two additional approaches: the analysis of mitochondrial protein abundance by Western blotting, and flow cytometry analyses of mitochondrial mass. Biochemical analyses were carried out in HEK293 cells, a mitochondria-rich cell model previously used to characterise the presence and the functions of AURKA at mitochondria (Bertolin et al, 2018). In Western blotting analyses of total protein fractions, we monitored the abundance of the OMM protein TOMM22 and of the IMM protein TIMM50. Consistent with confocal microscopy data, a significant loss of both markers was observed in cells overexpressing AURKA (Fig 1B).

Similarly, we evaluated the loss of mitochondrial mass by flow cytometry taking advantage of the polarisation-insensitive dye MitoTracker Green. When overexpressing AURKA in HEK293 cells, the mean fluorescence intensity of MitoTracker Green decreased by nearly 50\% (Fig 1C). Concomitantly with a decrease in MitoTracker Green fluorescence intensity, the polarisation-sensitive dye TMRM failed to accumulate at mitochondria under these conditions (Fig S1C). This indicates that mitochondria of cells overexpressing AURKA undergo a loss of mitochondrial membrane potential.

As the loss of mitochondrial mass is commonly associated with the autophagic elimination of mitochondria-mitophagy-(Klionsky et al, 2016), we wanted to explore whether AURKA plays a role in this turnover pathway. We first monitored the activation of the autophagic marker MAP1LC3 in HEK293 cells. Measured as the conversion of MAP1LC3 I into II, this modification is a commonly used readout to assess activation of autophagy (Klionsky et al, 2016). We observed an increase in MAP1LC3II only when AURKA was overexpressed (Fig 1D). However, we sought to determine whether MAP1LC3 activation was a sign of bulk autophagy or of mitophagy. Using confocal microscopy, we evaluated the proportion of mitochondria colocalising with MAP1LC3-positive vesicles in MCF7 cells. The proximity of mitochondria and MAP1LC3 or the lysosomal marker LAMP1 was observed upon the overexpression of AURKA, but not in the presence of AURKA $\triangle$ Nter or in control cells (Fig 1E). We further confirmed these results in the fruit fly, a model that allows the comparison of the effects of a physiological expression of AURKA to its loss-of-function or gain-offunction. We quantified the number of Atg8 dots, the fly orthologue of MAP1LC3, juxtaposed to mitoGFP-positive mitochondria, and normalised to the total number of Atg8 dots (Fig S1D). Quantifications were carried out in the fly notum, a monolayer of epithelial cells. Again, we observed an increased number of Atg8 dots on mitochondria only when AURKA was overexpressed, and this was not observed in any other condition.

Last, we reasoned that an increased mitophagy could be induced by an alteration of the mitochondrial biogenesis/turnover equilibrium. We first analysed mitochondrial mass in the presence of increasing quantities of AURKA in non-tumorigenic human mammary epithelial cells (HMLE) (Mani et al, 2008) (Fig S2). Mitochondrial loss reached its peak when cells were nucleofected with $200 \mathrm{ng}$ of a vector encoding AURKA-GFP. However, cells nucleofected with 500 ng of AURKA-GFP showed a more heterogeneous behaviour, with some cells undergoing mitochondrial loss and some showing a 
quantity of mitochondria similar to control cells. HMLE cells nucleofected with $1 \mu \mathrm{g}$ of AURKA-GFP displayed a mitochondrial mass similar to those of control cells, raising the possibility that a complementary biogenesis pathway is activated to overcome mitochondrial loss under these conditions. These data indicate a potential co-regulation of mitochondrial turnover and biogenesis programs, according to the quantity of AURKA present in the cell. We then verified whether such changes could be reflected by AURKAdependent alterations of mitochondrial biogenesis factors. The relative abundance of the nuclear-encoded protein succinate dehydrogenase [ubiquinone] flavoprotein subunit (SDHA), or the PGC1A transcription factor was not altered in cells overexpressing AURKA compared with cells transfected with an empty vector or AURKA $\triangle$ Nter (Fig 1F). However, the abundance of the mtDNAencoded protein MT-CO1 was increased upon overexpression of AURKA (Fig 1F) (Bertolin et al, 2018). Although we could not retrieve a global effect of the kinase on proteins orchestrating organelle biogenesis, it is possible that MT-CO1 is a preferential partner of AURKA in the regulation of mitochondrial functions.

These data indicate that the overexpression of AURKA induces the recruitment of MAP1LC3 to mitochondria, together with mitochondrial depolarisation and a loss of mitochondrial mass.

\section{AURKA overexpression leads to lysosomal accumulation and to the activation of mitophagy-related probes}

As our results indicate the presence of mitophagy-related features in cells overexpressing AURKA, we then used transmission electron microscopy to better visualise this turnover. In HEK293 cells subjected to TEM analyses after the overexpression of AURKA, we observed that lysosomes were more abundant than in control cells or in cells where the kinase was silenced (Fig 2A). A nearly twofold increase in the lysosomal content was confirmed in live HEK293 cells incubated with LysoTracker Red, a fluorescent probe used to measure lysosomal mass (Fig 2B). The overexpression of the cytosolic-only AURKA $\triangle$ Nter variant did not affect lysosomal mass. In addition, TEM images revealed that lysosomes (Fig 2A, right panel, magenta arrows) were proximal to fragmented mitochondria $(m)$, which are known to be preferential targets for degradation by mitophagy (Twig \& Shirihai, 2011). We then evaluated whether this increase in lysosomal content was a mere accumulation, or one of the readouts of activated, mitochondrial-related autophagy. To this end, we evaluated the red/green fluorescence ratio of a mitochondrially targeted GFP-mCherry tandem (hereby, mitoTandem). This tool relies on the quenching of the GFP fluorophore inside lysosomes because of their acidic $\mathrm{pH}$, whereas the mCherry fluorophore is insensitive to the $\mathrm{pH}$ properties of the surrounding environment.mitoTandem has previously been used to estimate the presence of mitochondrial-related autophagy (Princely Abudu et al, 2019). In control cells not overexpressing AURKA or in cells overexpressing AURKA $\triangle N$ ter, the mCherry/GFP integrated density ratio was close to one, indicating no significant differences in the fluorescence intensities of both fluorophores (Fig S3A). Conversely, the mCherry/GFP ratio was increased in the presence of overexpressed AURKA, potentially because of the quenching of GFP in an acidic environment and suggesting the presence of mitochondriarelated autophagy.
To further verify the activation of mitophagy in cells overexpressing AURKA, we investigated whether AURKA directly interacts with selective components of the autophagic pathway, providing a potentially direct link to the loss of mitochondrial mass and the increase in the number of lysosomes in AURKA-overexpressing cells. To search for weak and/ or potentially transient protein-protein interactions between AURKA and the molecular players of autophagy, we used Förster's Resonance Energy Transfer (FRET)/Fluorescence Lifetime Imaging Microscopy (FLIM) (Padilla-Parra et al, 2008; Leray et al, 2013). FRET was represented as $\Delta$ Lifetime, which is the net difference between the lifetime of the donor-only construct and the donor in the presence of the acceptor. A positive $\Delta$ Lifetime is indicative of FRET, and therefore of a physical interaction between two proteins. We previously used $\Delta$ Lifetime as a convenient way to illustrate molecular interactions (Bertolin et al, 2019). FRET/FLIM analyses revealed that overexpressed AURKA is in close proximity ( $<10 \mathrm{~nm}$, consistent with molecular interactions) with MAP1LC3 and with the lysosomal protein LAMP1 in MCF7 cells (Fig 2C). Accordingly, no interaction of AURKA $\triangle$ Nter with MAP1LC3 was detected by FRET/FLIM (Fig 2D). This differential effect was not due to the expression levels of the fluorescent proteins used, as the $\triangle$ Lifetime values of AURKA-GFP and AURKA $\triangle$ Nter-GFP expressed with mCherry-MAP1LC3 showed overall similar red/green ratio levels (Fig 2E). This also indicates that AURKA must be imported into mitochondria (Bertolin et al, 2018; Grant et al, 2018) and potentially processed into this compartment to come into contact with autophagic markers during the process of mitochondrial elimination. To corroborate FRET/FLIM analyses, we used the fluorescent mCherry-GFP MAP1LC3 tandem to follow autophagosomal maturation in cells overexpressing AURKA (Tresse et al, 2010). Similarly to mitoTandem, yellow MAP1LC3 puncta indicate the presence of autophagosomes in a non-acidic environment, whereas mCherryonly puncta are the consequence of GFP quenching and indicate the presence of autolysosomes. In cells overexpressing AURKA, both autophagosomes and autolysosomes were present (Fig S3B), substantiating the capacity of AURKA to interact with early (MAP1LC3) and late (LAMP1) markers of the autophagic pathway (Fig 2C). On the contrary, in control cells we did not observe a massive autophagosomal or autolysosomal activation (Fig S3B).

Overall, our data describe the activation of autophagy-specific markers on mitochondria and of mitophagy-specific probes in cells overexpressing AURKA. As this strongly suggests ongoing mitophagy, we next evaluated the presence of key events known to occur in the mitophagy cascade.

\section{The overexpression of AURKA independently induces proteasome-dependent disappearance of OMM markers and autophagy-dependent IMM digestion}

During mitophagy, mitochondrial elimination requires the proteasomedependent degradation of the OMM (Chan et al, 2011; Yoshii et al, 2011; Wei et al, 2017; Di Rita et al, 2018). We thus explored whether the loss of mitochondrial inner markers occurring in MCF7 cells overexpressing AURKA passes first by a loss of OMM markers. To this end, we monitored the disappearance of the OMM marker TOMM22 in the presence or absence of the proteasomal inhibitor MG132. In immunofluorescence analyses, TOMM22 levels significantly decreased in cells overexpressing AURKA (Fig 3A, upper panel), and the treatment with MG132 rescued its 

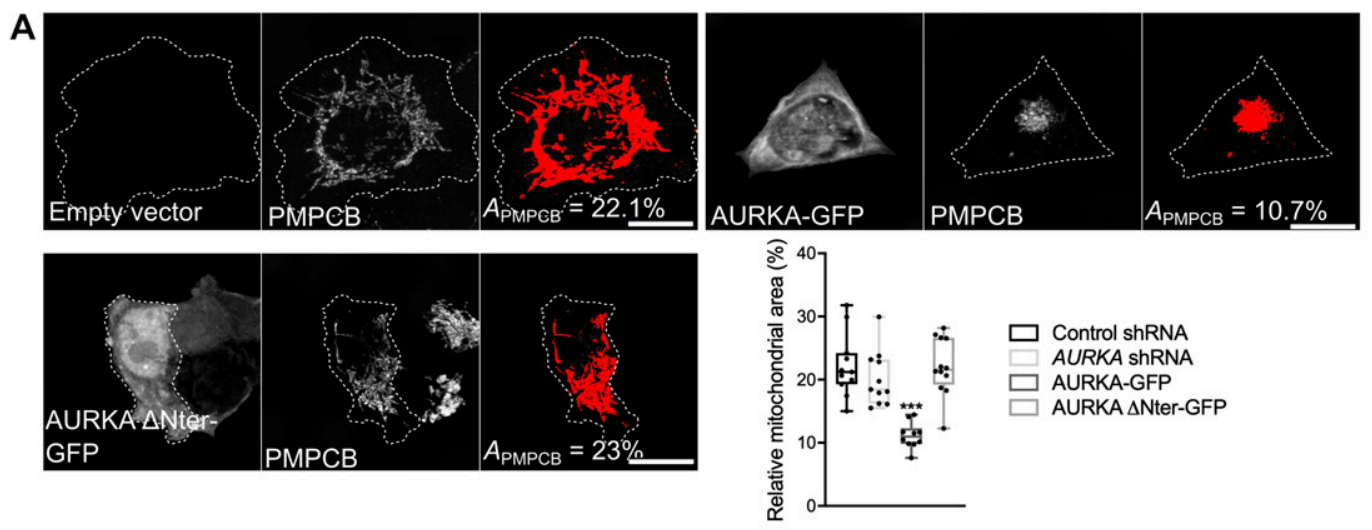

B
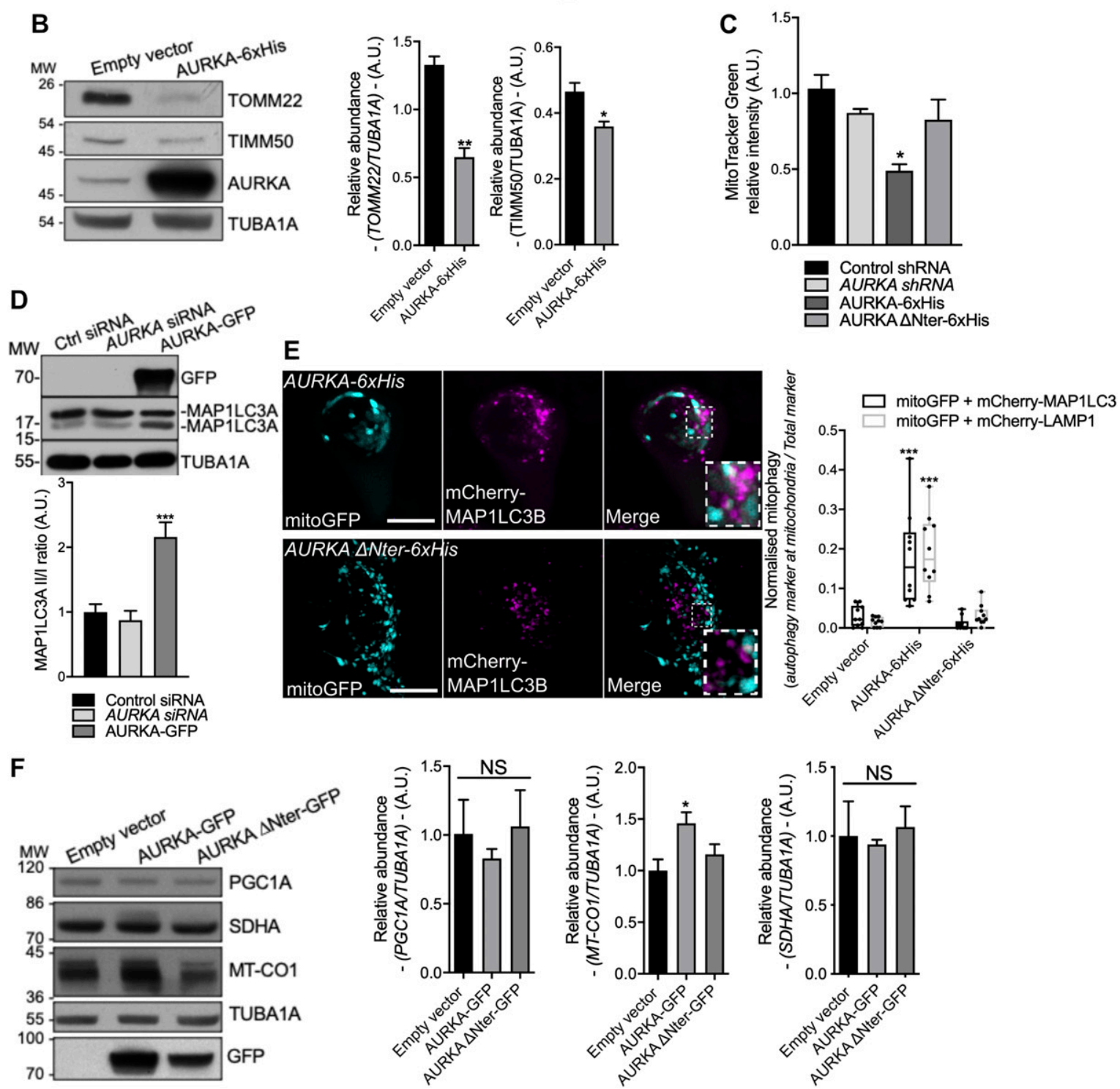

Figure 1. Mitochondria are eliminated by mitophagy in cells overexpressing AURKA.

(A) Loss of PMPCB staining (threshold mask and corresponding quantification) in MCF7 cells transfected with an empty vector, AURKA-GFP or AURKA $\triangle$ Nter-GFP. A = mitochondrial area normalised against total cell area (\%). $n=10$ cells per condition from one representative experiment (of three). (B) Representative immunoblot and quantification of the normalised abundance of the outer mitochondrial membrane marker TOMM22 and of the inner mitochondrial membrane marker TIMM50 in total lysates of HEK293 cells transfected as indicated. Loading control: TUBA1A. $n=3$ independent experiments. (C) Relative MitoTracker Green FM fluorescence measured by flow cytometry on HEK293 cells transfected as indicated. $n=3$ independent experiments with at least 30,000 cells per condition quantified. (D) Representative 
loss of (Fig 3A, lower panel). We obtained similar results using Western blotting and monitoring another OMM marker-MFN2-(Fig 3B). This suggests that the OMM markers are lost in a proteasome-dependent manner when AURKA is overexpressed.

The elimination of IMM proteins was previously shown to depend on the autophagy machinery (Yoshii et al, 2011). We then explored whether the mitochondrial innermost compartments undergo elimination through the autophagy pathway in AURKA-overexpressing cells. To this end, we used immunofluorescence approaches to quantify the loss of mitochondrial-processing peptidase subunit beta (PMPCB), in cells overexpressing AURKA and in the presence or absence of the early autophagic inhibitor 3-methyladenine, or of the late autophagic inhibitor bafilomycin A (Fig 3C). Both inhibitors rescued the loss of PMPCB staining observed in cells treated with DMSO, as the mitochondrial mass observed in the presence of these two compounds is similar to the one of cells transfected with a control vector and not showing signs of mitochondrial loss. Again, similar results were obtained using Western blotting approaches and with another IMM marker-TIMM50 (Fig 3B). We also noticed the existence of PMPCBpositive/TOM22-negative mitochondria in cells overexpressing AURKA (Fig 3D), but not in cells expressing an AURKA $\triangle$ Nter construct. These observations further suggest that the degradation of the OMM occurs before the digestion of the IMM/matrix compartments.

We then explored whether OMM rupture is necessary for the elimination of IMM/matrix markers. The loss of PMPCB and of TIMM50 were not restored when cells overexpressing AURKA were treated with MG132 (Figs 3B and S4A). This indicates that the loss of OMM markers is not required for IMM/matrix degradation. As an additional way to explore whether AURKA-dependent IMM/matrix loss requires the activity of the proteasome, we analysed whether AURKA is still capable of interacting with MAP1LC3 upon proteasome inhibition. When the loss of OMM integrity is prevented by the addition of MG132, AURKA and MAP1LC3 still show a positive $\triangle$ Lifetime in FRET / FLIM analyses (Fig S4B). This was not due to a side effect of MG132, as the addition of the drug did not modify the lifetime of AURKA-GFP in the absence of the acceptor. Therefore, our results indicate that AURKA and MAP1LC3 interact in a comparable manner in the presence and in the absence of OMM integrity. Furthermore, we observed that the elimination of the OMM proteins TOMM22 and MFN2 did not require the autophagy degradation system (Figs $3 \mathrm{~B}$ and $\mathrm{S} 4 \mathrm{C}$ ). This further supports the dissociation of $\mathrm{OMM}$ and $\mathrm{IMM} /$ matrix degradation pathways in AURKA-overexpressing cells.

Overall, our data indicate that the overexpression of AURKA recapitulates two features observed in other paradigms of mitophagy, that is, the proteasome-dependent loss of OMM markers, and an autophagy-dependent IMM/matrix degradation. We also provide evidence that a loss of OMM integrity is not a prerequisite for AURKA-dependent mitochondrial mass loss, as $\mathrm{IMM} /$ matrix markers can be digested to the same extent in the presence or in the absence of OMM ones.

\section{Mitophagy in AURKA-overexpressing cells is independent of PARK2/Parkin}

Given that cells overexpressing AURKA have a sequential proteolysis similar to that observed in PARK2/Parkin-dependent mitophagy (Chan et al, 2011; Yoshii et al, 2011), we evaluated whether AURKA induces mitochondrial mass loss within this pathway or outside of it. We first evaluated whether PARK2/Parkin is an AURKA interactor. Neither PARK2/Parkin nor its partner PINK1 were retrieved in the proteomics data constituting the interactome of AURKA at interphase in HEK293 cells (Bertolin et al, 2018). However, failure in detecting these interactions could potentially reflect dynamic interactions taking place only when mitophagy is activated, and not when AURKA regulates mitochondrial morphology or ATP production (Bertolin et al, 2018). As the recruitment of PARK2/ Parkin to mitochondria is among the first events in the degradation programme orchestrated by PARK2/Parkin and PINK1 (Pickrell \& Youle, 2015), we used this as a functional readout of mitophagy activation. In cells overexpressing AURKA, PARK2/Parkin-GFP remained largely cytosolic and no colocalisation was observed between PARK2/Parkin and the mitochondrial marker PMPCB (Fig 4A, left panels). On the contrary, PARK2/Parkin was entirely mitochondrial in cells treated with the mitochondrial uncoupler carbonyl cyanide 3-chlorophenylhydrazone (CCCP) (Fig 4A, right panels) (Narendra et al, 2008). These results indicate that although there is a visible loss in mitochondrial mass, no initiation of PARK2/ Parkin-dependent mitophagy occurs in cells overexpressing AURKA. To corroborate these results, we explored whether the loss of mitochondrial mass observed in cells overexpressing AURKA depends on the presence of PARK2/Parkin. siRNA-mediated downregulation of PARK2 in MCF7 cells overexpressing AURKA did not rescue mitochondrial elimination (Fig $4 \mathrm{~B}$ ). As recently done for STX17-dependent mitophagy, which was shown to be independent of PARK2/Parkin (Xian et al, 2019), we found that PARK2/Parkin was not expressed when the overexpression of AURKA induced mitochondrial clearance, although we observed the concomitant loss of the IMM marker MT-CO2 (Fig 4C). We also tested whether the absence of AURKA could alter PARK2/Parkin-dependent mitophagy in cells where PARK2/Parkin is overexpressed and mitochondria are

immunoblot of the autophagy marker MAP1LC3 and quantification of the MAP1LC3II/I ratio in total lysates of HEK293 cells transfected as indicated. $n=3$ independent experiments. (E) (Left) Immunofluorescence micrographs of MCF7 cells overexpressing AURKA 6xHis (top panels) or AURKA $\triangle N$ ter-6xHis (bottom panels), and transfected with a mitochondrially targeted GFP (mitoGFP) and mCherry-MAP1LC3B constructs. Inset: higher magnification of the dotted area. Right: corresponding colocalisation coefficient between mitoGFP and mCherry-MAP1LC3B or LAMP1 in the indicated conditions. $n=10$ cells per condition from one representative experiment (of three). (F) Representative immunoblot and quantification of the normalised abundance of the mitochondrial biogenesis factors PGC1A, MT-CO1, and SDHA in total lysates of HEK293 cells transfected as indicated. Loading control: TUBA1A. $n=3$ independent experiments. Scale bar: $10 \mu \mathrm{m}$. (A, C) Data represent means \pm SEM, unless in $(\mathrm{A}, \mathrm{C})$ where they extend from min to max. (A, B, C, D, E, F) ${ }^{\star} P<0.05$, ${ }^{* *} P<0.01$, ${ }^{* *} P<0.001$ compared with the corresponding “Control” (A, B, E), "AURKA" (C), or “Empty vector" conditions (D, F). NS, not significant; A.U., arbitrary units.

Source data are available for this figure. 
A

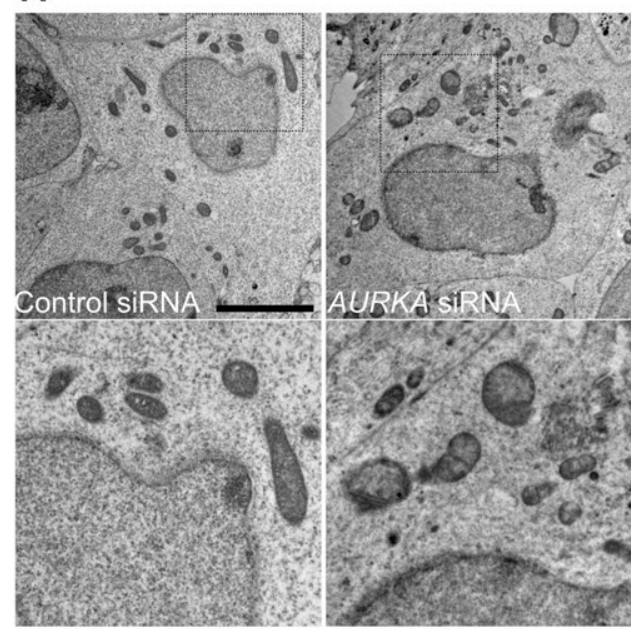

C
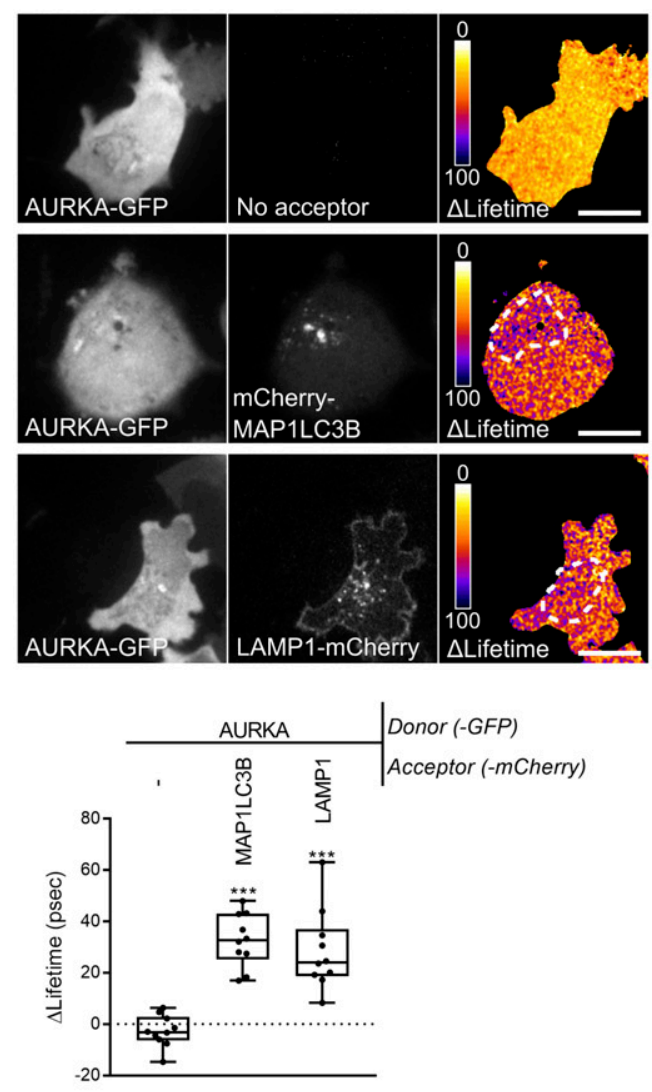

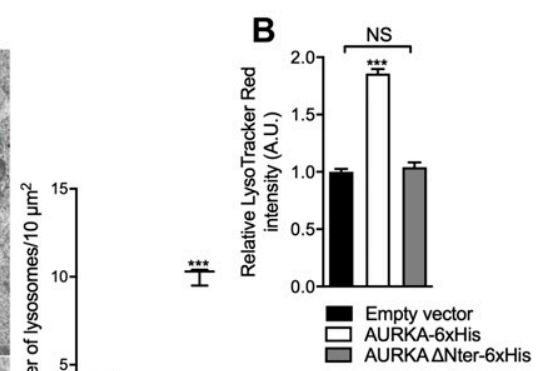

D
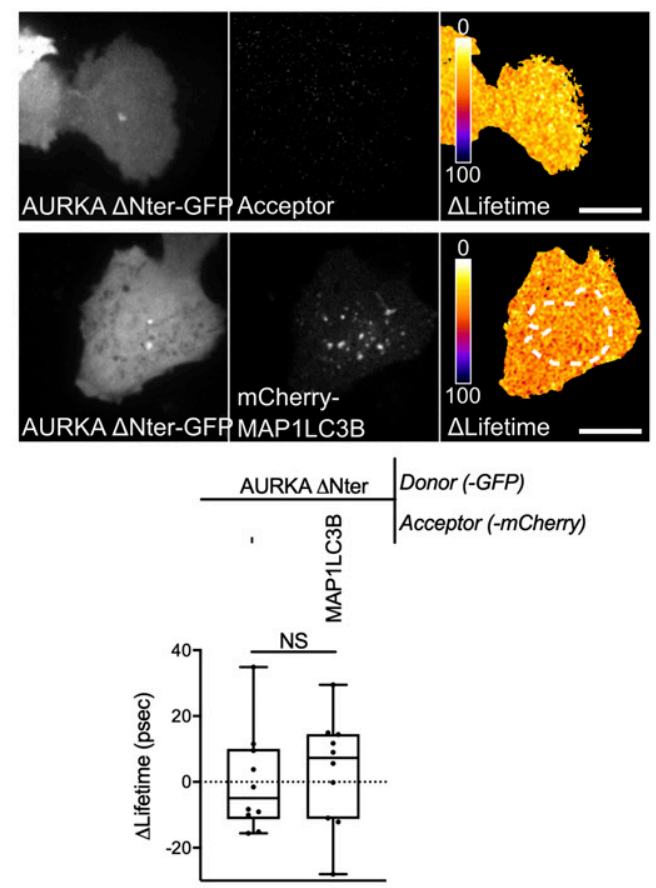

E

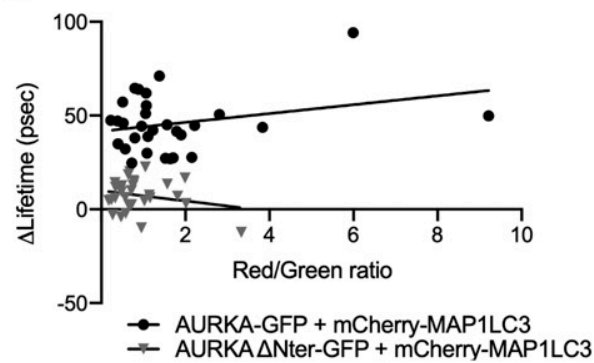

Figure 2. The autophagic machinery is activated and it interacts with overexpressed AURKA.

(A) Left: HEK293 cells imaged by transmission electron microscopy. Lower panels correspond to the magnified dotted area. Cells were transfected with control- or AURKA-specific siRNAs, or with an AURKA-GFP vector (AURKA O/E). $\mathrm{m}=$ mitochondria; magenta arrows = lysosomes. (Right) Quantification of lysosomal number per $10 \mu \mathrm{m}^{2}$ of cell surface. $n=20$ images per condition from two independent experiments. Scale bar: $1 \mu \mathrm{m}$. (B) Relative LysoTracker Red fluorescence measured by flow cytometry on HEK293 cells transfected as indicated. $n=3$ independent experiments with at least 30,000 cells per condition quantified. Data represent means \pm SEM, A.U., arbitrary units. (C, D) Förster's Resonance Energy Transfer by Fluorescence Lifetime Imaging Microscopy analyses on MCF7 cells expressing AURKA-GFP (C) or AURKA $\triangle$ Nter-GFP (D) 
depolarised with CCCP (Narendra et al, 2008). AURKA knockdown does not perturb the mitochondrial mass loss induced by CCCPmediated depolarisation in cells overexpressing PARK2/Parkin (Fig 4D). These results indicate that AURKA induces organelle clearance in a PARK2/Parkin-independent fashion, and that the two clearance pathways are independent.

\section{AURKA interacts with PHB2 during mitophagy}

PHB2 is an integral component of the IMM, and together with the highly homologous protein PHB it forms the Prohibitin heterodimeric complex (Merkwirth et al, 2008). We previously identified the Prohibitin complex (PHB/PHB2) as one of the most prominent interactors of AURKA at mitochondria by quantitative proteomics coupled to MS/MS (Bertolin et al, 2018). This interaction was confirmed by FRET/FLIM analyses in MFC7 cells, where we observed significant AURKA-GFP $\triangle$ Lifetime variations in zones where PHB-mCherry or PHB2-mCherry were present (Fig 5A). These interactions were not due to the tight environment of the IMM, as no FRET was retrieved with calcium uniporter protein, mitochondrial (MCU) or MIC60, two abundant IMM markers (Figs 5B and S5A). Given the key role of PHB2, but not of PHB, in mitochondrial turnover (Wei et al, 2017), we focused on the interaction between AURKA and PHB2. The depletion of PARK2 by siRNA-mediated gene down-regulation did not abolish the interaction between AURKA and PHB2, further confirming that AURKAdependent mitochondrial loss is independent of PARK2/Parkin (Figs $5 \mathrm{C}$ and $\mathrm{S} 5 \mathrm{~B}$ ). We then examined whether the interaction between AURKA and PHB2 is due to the import of AURKA into mitochondria (Bertolin et al, 2018), or to a physical proximity between the two proteins after the disappearance of OMM markers (Fig 3C). To discriminate between these two possibilities, we first tested the interaction between the non-importable AURKA $\triangle$ Nter with PHB2 by FRET/FLIM. With this construct, we observed that the AURKA/PHB2 proximity is abolished (Fig 5D). Then, we analysed the capacity of AURKA to interact with PHB2 in the presence of MG132, which blocks OMM rupture when AURKA is overexpressed. In this case, FRET/FLIM analyses indicated that the physical interaction between AURKA and PHB2 is maintained (Fig S5C). These results show that the disappearance of OMM markers not necessary for AURKA and PHB2 to interact, whereas the import of the kinase into mitochondria is required for its interaction with the mitophagy receptor.

We then verified the functional consequences of this interaction, by exploring whether overexpressed AURKA induced mitochondrial mass loss in a PHB2-dependent manner. We knocked-down PHB2 by siRNA-mediated gene down-regulation, and we quantified the loss of the mitochondrial marker PMPCB upon the overexpression of AURKA (Fig 5E). In cells overexpressing AURKA and transfected with a control siRNA or a siRNA against PHB, the abundance of PMPCB was 50\% less than in cells without exogenous AURKA. On the contrary, the loss of PMPCB was significantly mitigated in cells overexpressing AURKA and depleted of PHB2, indicating that PHB2 is essential for AURKA-dependent mitophagy. Accordingly, Western blotting analyses of T47D cells which physiologically overexpress AURKA (Bertolin et al, 2018) showed that the down-regulation of either AURKA or of $P H B 2$ in this model is sufficient to increase the abundance of TOMM22 and of TIMM50 (Fig 5F). These results are in accordance with data obtained with confocal microscopy approaches (Fig S1B), and suggest that the two proteins trigger mitophagy within a single molecular pathway.

Together, these results show that AURKA interacts with PHB2 and that this interaction is mandatory to trigger mitochondrial elimination.

\section{AURKA triggers mitophagy via the phosphorylation of PHB2 on Ser39}

Given that AURKA interacts both with PHB2 and with MAP1LC3 in the context of mitochondrial elimination, we hypothesised that the interaction between AURKA and MAP1LC3 at mitochondria was mediated by PHB2. FRET/FLIM analyses performed in PHB2-depleted cells revealed that AURKA and MAP1LC3 no longer interacted in the absence of the mitophagy receptor (Fig 6A). As the presence of PHB2 is mandatory to achieve mitochondrial elimination when AURKA is overexpressed, we explored whether PHB2 could be a substrate of AURKA. In silico simulations of AURKA-dependent phosphorylation using the GPS (Xue et al, 2005) or the PhosphoPICK (Patrick et al, 2016) methods, identified Ser39 as the only residue potentially phosphorylatable by AURKA which is conserved throughout evolution (Fig $6 \mathrm{~B})$. We therefore evaluated whether a mutant of PHB2 that cannot be phosphorylated on Ser39 retained its capacity to interact with AURKA. To this end, we mutated Ser39 into Ala and we analysed the capacity of this construct to interact with the kinase by FRET/FLIM analyses. AURKA still interacted with PHB2 when Ser39 was converted into an Ala, indicating that the phosphorylation of this residue is not necessary for AURKA and PHB2 to interact (Figs 6C and S6A). As shown above for non-mutated PHB2 (Fig 5A), the interactions between AURKA and PHB2 S39A or the phospho-mimetic S39D were abolished with the non-importable AURKA $\triangle$ Nter (Figs 6C and S6B).

Although the phosphorylation of this residue seems not to be required for AURKA to directly interact with PHB2, its conservation throughout evolution led us to hypothesise that it could still play a role in AURKA-dependent mitophagy. Therefore, we analysed the loss of PMPCB in cells overexpressing AURKA and PHB2, its S39A or S39D counterparts by confocal microscopy. We observed that the expression of PHB2 S39A abolished AURKA-dependent mitochondrial loss, whereas its phospho-mimicking mutant restored it (Figs 6D and S6C).

Data from in silico simulations and functional mutants indicate that Ser39 is a putative phosphorylation site for AURKA on PHB2. Our results show that the interaction between AURKA and MAP1LC3 depends on PHB2, and that the phosphorylation of Ser39 on PHB2 is required for mitophagy when the kinase is overexpressed.

alone or together with mCherry-MAP1LC3B or LAMP1-mCherry. Dotted area: autophagosome/autolysosome-rich areas. Pseudocolour scale: pixel-by-pixel $\triangle$ Lifetime. Lower panels: corresponding $\Delta$ Lifetime quantifications in the dotted area. $n=10$ cells per condition of one representative experiment (of three). (E) Graphical representation of AURKA-GFP or AURKA $\triangle$ Nter-GFP $\triangle$ Lifetime values in the presence of mCherry MAP1LC3, plotted as a function of the red $/$ green intensity ratio. $n=30$ cells per condition from three independent experiments. Scale bar: $10 \mu \mathrm{m}$. Data extend from minimum to maximum, unless where indicated. (A, B, C, D) $\star \star \star P<0.001$ against the "Control siRNA" (A), the "Empty vector" (B), or the "AURKA-No acceptor" (C, D) conditions. NS, not significant. 

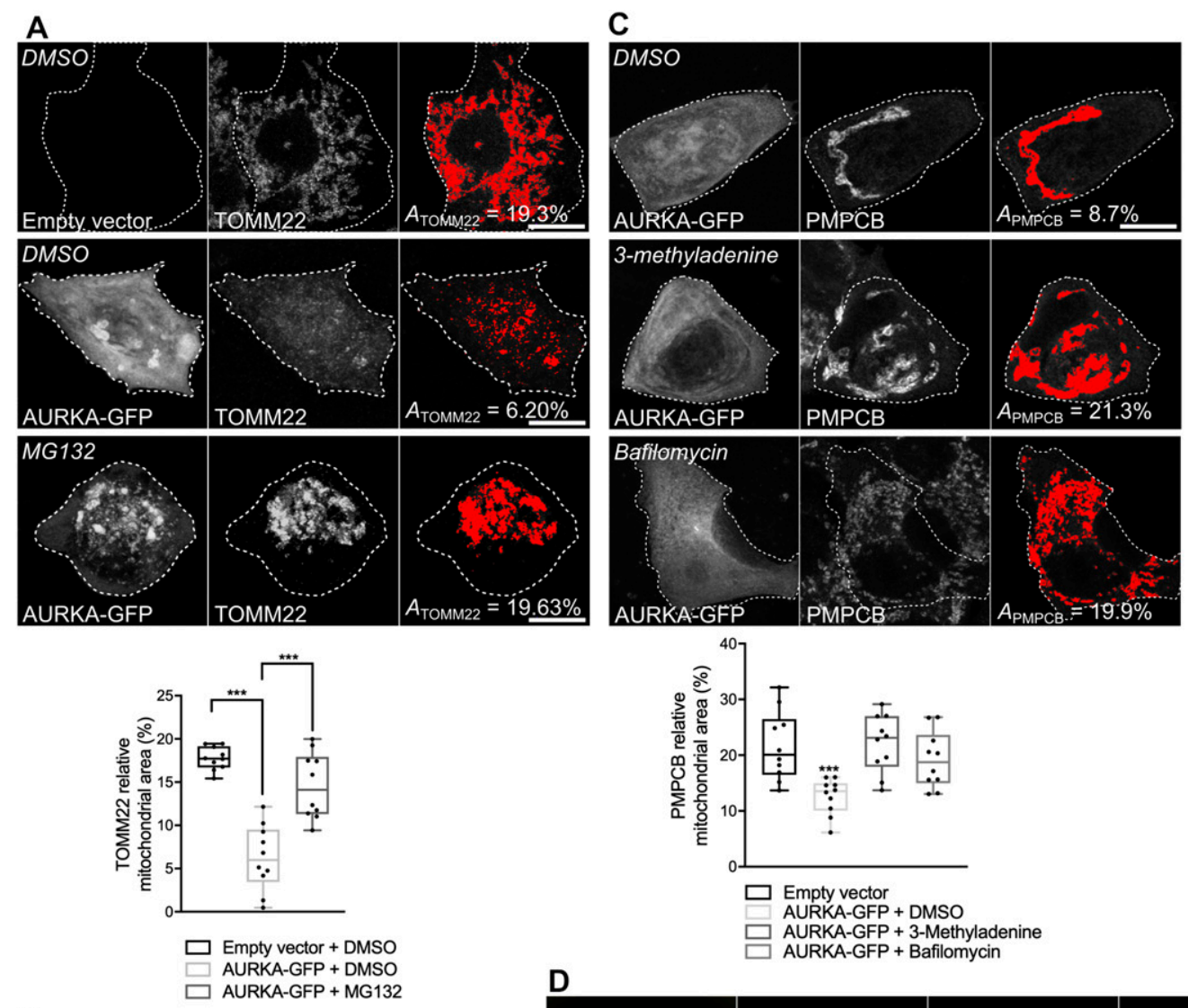

B
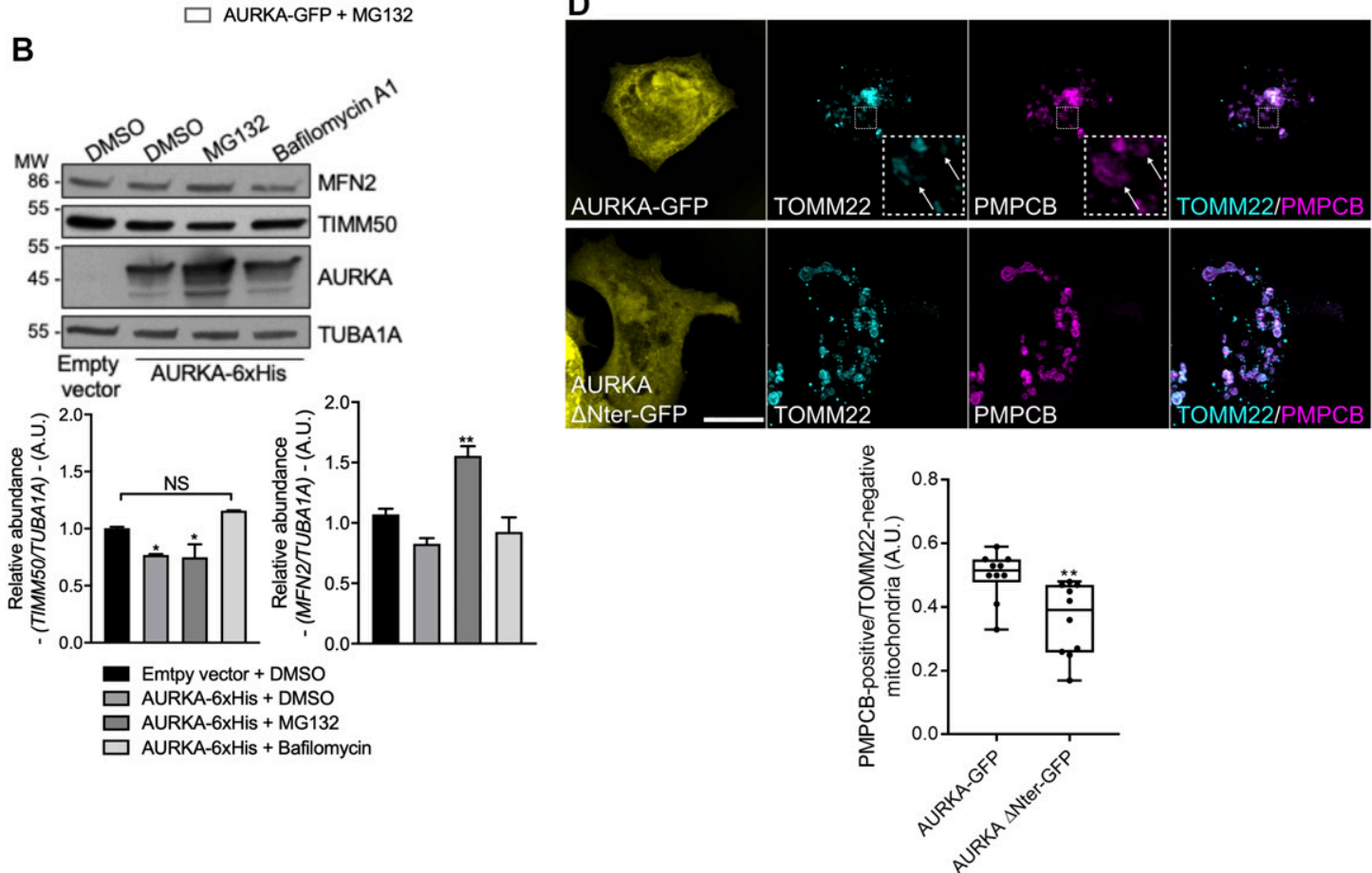

Figure 3. The outer mitochondrial membrane is ruptured in a proteasome-dependent manner when AURKA is overexpressed.

(A) Loss of TOMM22 staining (threshold mask and corresponding quantification) in MCF7 cells transfected with an empty vector or with AURKA-GFP, and treated with DMSO or with the proteasome inhibitor MG132. (B) Representative immunoblot and quantification of the normalised abundance of the outer mitochondrial membrane marker MFN2 and of the inner mitochondrial membrane marker TIMM50 in total lysates of HEK293 cells transfected with AURKA-6xHis or an empty vector, and treated as indicated. Loading control: TUBA1A. $n=3$ independent experiments. (C) Loss of PMPCB staining (threshold mask and corresponding quantification) in MCF7 cells transfected with as indicated, and treated with DMSO or with autophagy inhibitors 3-methyladenine (3-MA) or bafilomycin A1. (D) Representative micrographs and 


\section{The phosphorylation of PHB2 on Ser39 is mandatory to create a functional AURKA-MAP1LC3-PHB2 tripartite complex}

A PHB2 S39A phospho-inactive mutant impairs mitophagy without perturbing the AURKA/PHB2 protein-protein interaction. We then analysed whether PHB2 Ser39 phosphorylation was necessary for AURKA to interact with MAP1LC3. Again, we used FRET/FLIM analyses to sense the interaction between AURKA and MAP1LC3 in the presence of PHB2, PHB2 S39A, and PHB2 S39D. No difference was observed in the capacity of AURKA to interact with MAP1LC3, regardless of the PHB2 variant used (Fig 7A). The overexpression of PHB2 was recently shown to induce mitophagy per se within the PINK1/PARK2-Parkin pathway (Yan et al, 2020). Given that AURKA interacts with MAP1LC3 regardless of the PHB2 variant used, we sought to verify whether we were triggering an additional mitophagy pathway by simply overexpressing PHB2. In this light, we used the lipidation rate of MAP1LC3 as a marker of mitophagy initiation in the presence of GFP-tagged PHB2, PHB2 S39A and S39D and in the absence of overexpressed AURKA. The overexpression of PHB2 or its variants did not induce alterations in the lipidation rate of MAP1LC3 compared with control cells, nor on the abundance of endogenous PHB2 (Fig 7B). Therefore, the overexpression of PHB2, PHB2 S39A, or PHB2 S39D do not trigger mitophagy activation under these conditions. This also reinforces the central role of overexpressed AURKA as a main activator of mitophagy and mitochondrial mass loss.

Recent evidence showed that PHB2 acts as the receptor of lipidated, active MAP1LC3 on the IMM during mitophagy (Wei et al, 2017). Accordingly, we explored whether we could retrieve a PHB2/MAP1LC3 interaction by FRET/FLIM. The positive $\triangle$ Lifetime between PHB2-GFP and mCherry-MAP1LC3B in cells overexpressing AURKA confirmed the existence of a tripartite complex constituted by these proteins (Fig 7C). We then assessed whether the phosphorylation of PHB2 on Ser39 was necessary for PHB2 and MAP1LC3 to interact. FRET/FLIM analyses revealed that the PHB2 S39A variant abolished the interaction between PHB2 and MAP1LC3, which was restored in the presence of the corresponding phospho-mimetic version, PHB2 S39D (Fig 7C).

These results indicate the existence of a tripartite complex where AURKA, PHB2 and MAP1LC3 interact. The phosphorylation of PHB2 on Ser39 is mandatory to build a functional tripartite complex, as PHB2 is incapable to perform FRET with MAP1LC3 if Ser39 is not phosphorylated. Failure in building a functional tripartite complex-by altering one of the proteinprotein proximities within the complex-abolishes mitophagy (Fig 6D).

\section{The PHB2 ligand xanthohumol inhibits mitophagy by impairing the tripartite complex, and it abolishes the AURKA-dependent ATP overproduction}

In the attempt of blocking AURKA-dependent mitophagy, we next looked for a pharmacological strategy to block the formation of a functional tripartite AURKA-MAP1LC3-PHB2 complex. The PHB2 ligand xanthohumol is a particularly interesting molecule in this context. It is a natural derivative of hop, which can block PHB2 functions in the nucleus (Yoshimaru et al, 2015). It can also block autophagy by targeting Valosin-Containing Protein and impairing the autophagosome-lysosome fusion (Sasazawa et al, 2012).

We first analysed whether a functional tripartite AURKA-MAP1LC3PHB2 complex was maintained upon treatment with xanthohumol. The incubation of cells with xanthohumol for $24 \mathrm{~h}$ did not abolish the interaction between AURKA and PHB2 (Fig 8A), nor the one between PHB2 and MAP1LC3B (Fig 8B). Of note, these interactions were significantly reinforced after xanthohumol treatment. Instead, FRET/FLIM analyses revealed that xanthohumol alters the proximity between AURKA and MAP1LC3B (Fig 8C). We previously observed that a dephosphorylated Ser39 in PHB2 destabilises protein-protein interactions within the tripartite complex (Fig 7C), and that a destabilised AURKA-MAP1LC3 interaction results in an impaired mitophagy (Fig 6A). We thus reasoned that the xanthohumol-mediated perturbation of protein-protein proximities within the functional tripartite complex could also be indicative of defective mitophagy. Therefore, we explored whether mitochondrial mass loss could be rescued in cells over-expressing AURKA and treated with this compound. As expected, we quantified a significant loss of PMPCB staining when cells overexpressing AURKA were treated with DMSO (Fig 8D). On the contrary, cells treated with xanthohumol showed an increase in PMPCB staining (Fig 8D), indicative of an increase in mitochondrial mass similar to that observed in control cells not overexpressing AURKA. Comparatively, treatment with a catalytic inhibitor of AURKA, MLN8237, abolished mitochondrial mass loss (Fig 8D). This reinforces our finding that AURKA kinase activity towards mitochondrial substrates is required for its role in mitophagy. These observations were also corroborated with Western blotting analyses illustrating the increase of the IMM marker TIMM50 after treating cells with xanthohumol or with MLN8237 (Figs 8E and S7). In addition, by using the MAP1LC3II / ratio to monitor the autophagic flux, we detected an increased MAP1LC3II accumulation and MAP1LC3II/I ratio in cells treated with xanthohumol (Fig 8E). These two parameters indicate that the autophagic flux is stalled, similarly to what described in previous reports (Sasazawa et al, 2012).

Last, we asked whether treatment with xanthohumol would revert the increased ATP production induced by AURKA overexpression (Bertolin et al, 2018). Therefore, we performed oxygen consumption rate (OCR) experiments, which measure the mitochondrial respiration capacity. As previously reported (Bertolin et al, 2018), cells overexpressing AURKA-GFP show an increased maximal respiration compared with cells expressing GFP alone (Fig $8 \mathrm{~F})$. This effect was abolished when cells overexpressing AURKA were treated with xanthohumol, whereas no effect of this compound was observed in control cells expressing GFP (Fig 8F).

corresponding analyses of MCF7 cells transfected with AURKA-GFP or AURKA $\triangle$ Nter-GFP and co-stained for TOMM22 and PMPCB. Insets: higher magnification of the dotted area. Arrows and quantification: TOMM22-negative/PMPCB-positive mitochondria. AURKA, TOMM22, and PMPCB were pseudocoloured yellow, cyan, and magenta, respectively. $A=$ mitochondrial area normalised against total cell area (\%). $n=10$ cells per condition from one representative experiment (of three). (B) Data extend from min to max unless in (B), where they represent mean \pm SEM. Scale bar: $10 \mu \mathrm{m}$. (A, B, C, D) ${ }^{\star} P<0.05,{ }^{* \star} P<0.01,{ }^{* * *} P<0.001$ against the "AURKA-GFP + DMSO" (A), the "Empty vector + DMSO" (B, C), or the "AURKA-GFP" (D) conditions. NS, not significant.

Source data are available for this figure. 
A
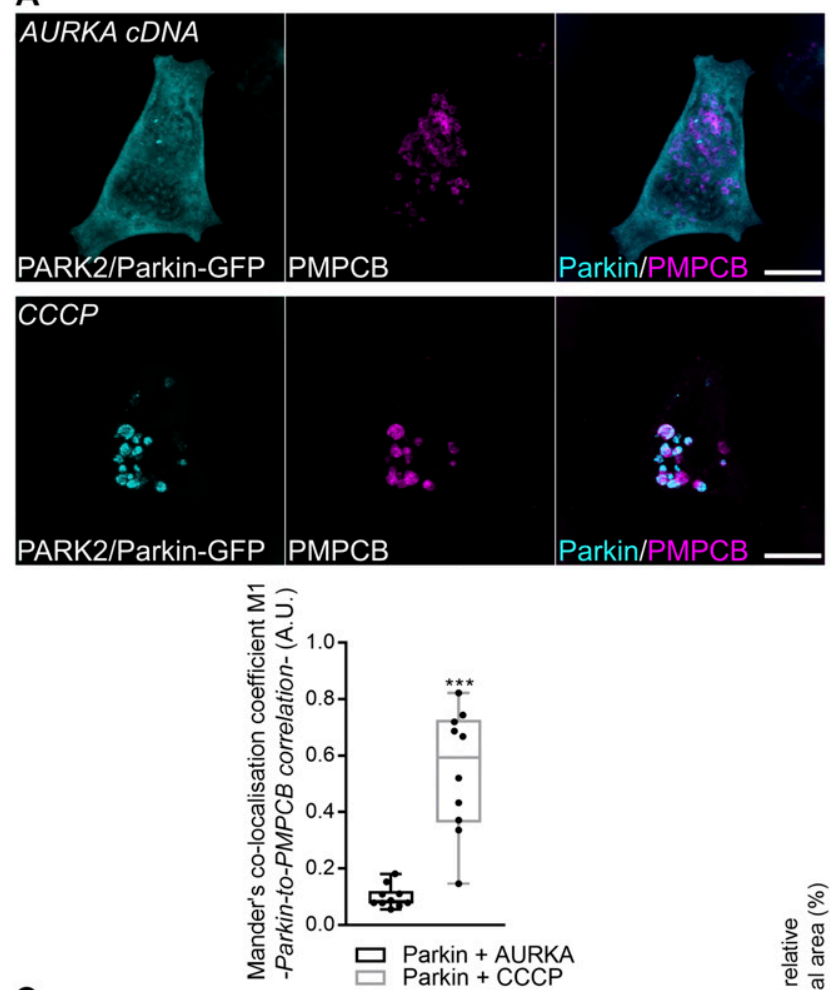

C
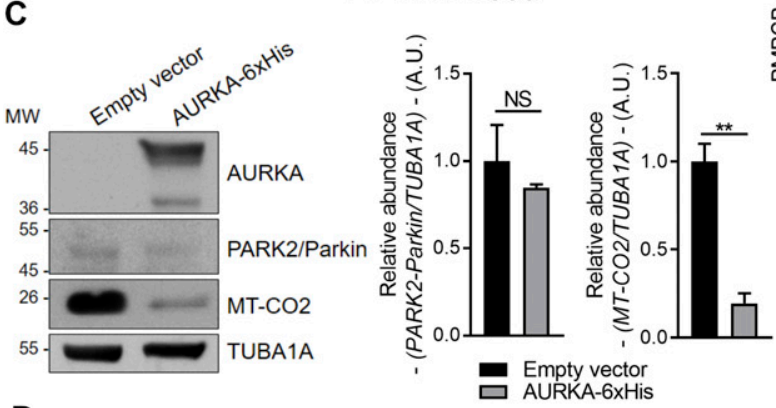

D

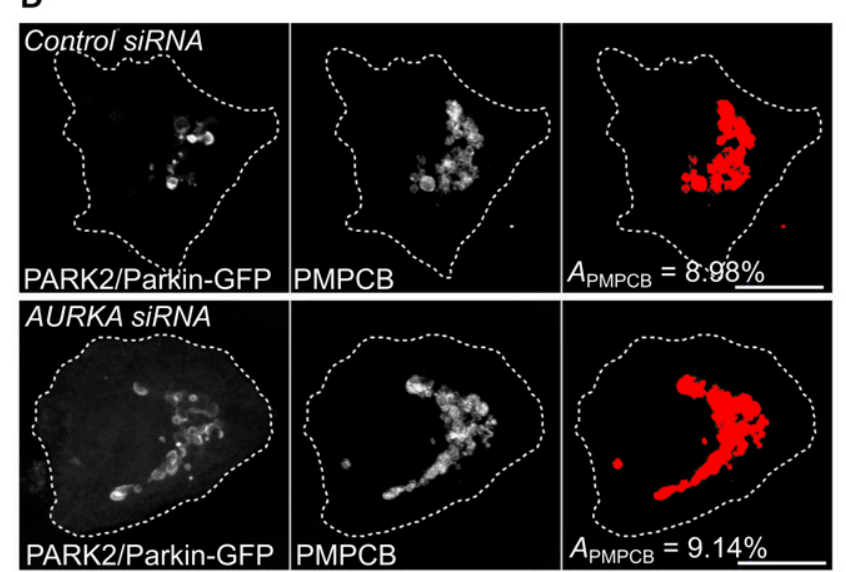

B

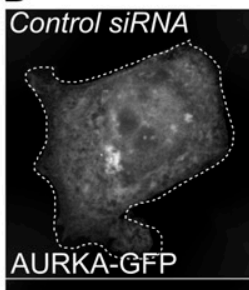

AURKA:GFP
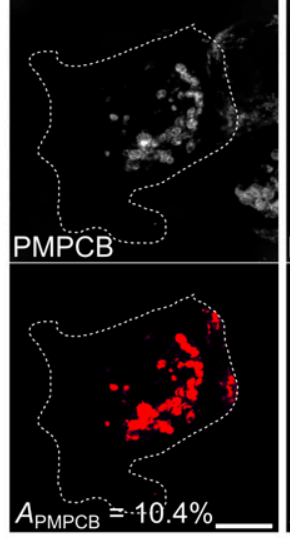

PMPCB

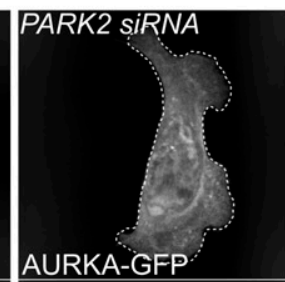

AURKA-GFP
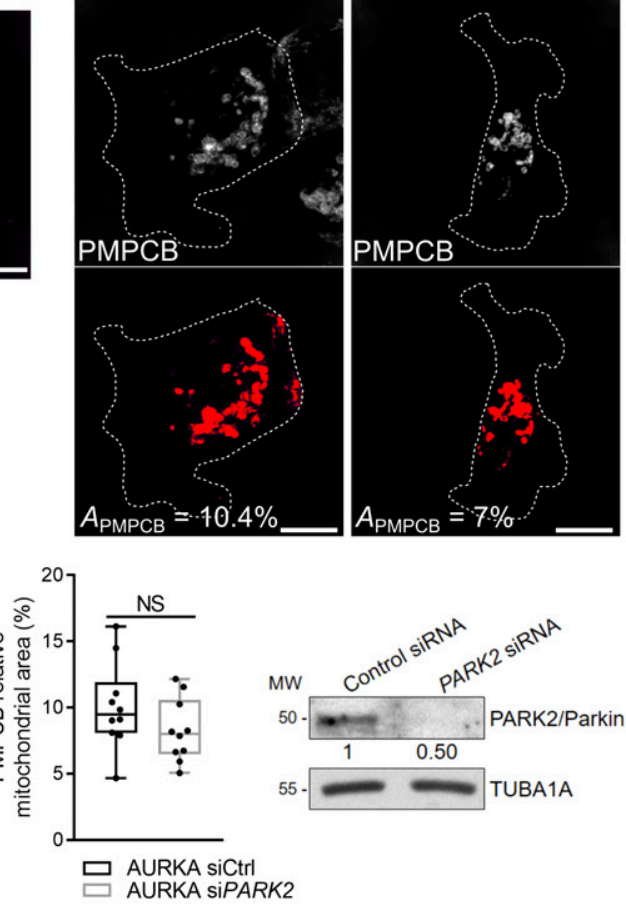

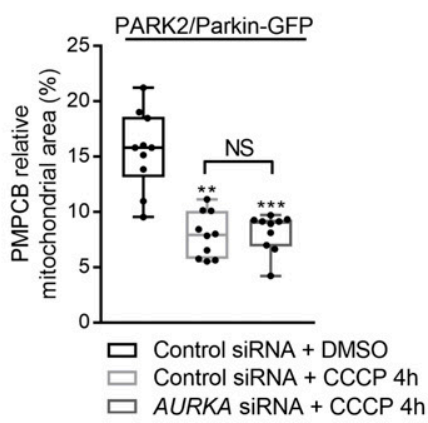

Figure 4. AURKA induces mitophagy independently of PARK2/Parkin.

Immunofluorescence micrographs of MCF7 cells co-expressing PARK2/Parkin-GFP and AURKA (left panels), or overexpressing PARK2/Parkin-GFP and treated with CCCP (right panels). Mitochondria were labelled with an anti-PMPCB antibody. PARK2/Parkin-GFP was pseudocoloured cyan, PMPCB was pseudocoloured magenta. Graph: corresponding colocalisation analyses (Mander's coefficient) between PARK2/Parkin-GFP and PMPCB. $n=10$ cells per condition from one representative experiment (of three). (B) LosS of PMPCB staining (threshold mask and corresponding quantification) in MCF7 cells co-transfected with AURKA-GFP and a control- or a PARK2-specific SiRNA. $A=$ mitochondrial area normalised against total cell area (\%). $n=10$ cells per condition from one representative experiment (of three). The efficiency of the PARK2- 
In conclusion, we here show that AURKA forms a mitophagyinducing tripartite complex with PHB2 and MAP1LC3 (Fig 9, left), upon the phosphorylation of PHB2 on Ser39. In addition, we show that AURKA-dependent mitophagy does not rely on the PARK2Parkin/PINK1 pathway. Last, abolishing protein-protein proximities within the tripartite complex creates a "dysfunctional" tripartite complex and blocks AURKA-dependent mitochondrial clearance, as observed with a Ser39A phospho-inactive mutant. Alternatively, the natural PHB2 ligand xanthohumol provides a pharmacological strategy to block the formation of a functional tripartite complex by altering the proximity between AURKA and MAP1LC3 (Fig 9, right). This ultimately rescues AURKA-dependent mitochondrial loss, and it abolishes the excessive ATP production detected under AURKA overexpression. Therefore, this corroborates the existence of a metabolic switch upon the overexpression of AURKA, and it further reinforces the link between the efficiency of mitophagy and the metabolic capacity of cancer cells.

\section{Discussion}

We here show that the multifunctional kinase AURKA plays a previously uncovered role at mitochondria, where it is a key actor of organelle clearance by mitophagy. This novel role further broadens our understanding of the molecular functions of AURKA at mitochondria (Kashatus et al, 2011; Bertolin et al, 2018; Grant et al, 2018). At the molecular level, we describe the interaction of AURKA with PHB2 as a key step in the mitophagy cascade. PHB2, which we previously identified as an interactor of AURKA in a proteomics screen (Bertolin et al, 2018), is phosphorylated on Ser39 and this allows the formation of a functional tripartite complex with MAP1LC3 triggering the elimination of mitochondria. In silico predictions and functional data from non-phosphorylatable Ser39 mutants also reveal that Ser39 is a putative AURKA phosphorylation site on PHB2.

The largest body of evidence concerning the pathways of mitophagy focuses on the PINK1/PARK2-Parkin molecular axis (Pickrell \& Youle, 2015). After nearly two decades of studies, there is general consensus that mitophagy pathways independent of PINK1/PARK2-Parkin exist, and that they play significant roles in specific pathological conditions as cancer, cardiac ischaemia and neurodegenerative disorders (reviewed in Villa et al [2018]). We here report that AURKA induces organelle turnover by interacting with MAP1LC3 on the mitophagy receptor PHB2, and without the contribution of PARK2/Parkin. Interestingly, this is the first evidence for a role of PHB2 outside of the PINK1-PARK2/Parkin pathway, which further extends the importance of this IMM receptor for various mitophagy paradigms. The finding that AURKA-dependent mitochondrial clearance does not require PARK2/Parkin opens several perspectives for future studies. Although proteasomedependent OMM rupture occurs in AURKA-overexpressing cells, it remains to be determined what E3 ubiquitin-protein ligase(s) can trigger this event. It is known that AURKA itself is targeted to the Ubiquitin-Proteasome system through Lys48- and Lys11-linked ubiquitin chains (Min et al, 2015). This occurs at mitotic exit, where the E3 Ubiquitin ligase $\mathrm{APC} / \mathrm{C}$ decreases the relative abundance of the kinase before a new cell cycle begins. Outside of mitosis and before cells re-enter the G2/M phase, other E3 ligases as CHFR (Yu et al, 2005) and the SCF complex members FBXW7, FBXL7, and FBXL2 (Fujii et al, 2006; Hagedorn et al, 2007; Coon et al, 2012; Chen et al, 2013) are thought to induce the turnover of AURKA. It has been proposed that only small subpopulations of AURKA are targeted to the proteasome from each of these $\mathrm{E} 3$ ligases (Lindon et al, 2016). Does a yet undiscovered mitochondrial E3 ubiquitin ligase trigger OMM rupture upon AURKA overexpression? Alternatively, is there a partner of AURKA on the OMM, which could serve as a platform regulating the rupture of this membrane in a kinasedependent manner? Interestingly, the AURKA domain targeted for APC/C-mediated ubiquitylation and degradation-located between residues 31-66 (Castro et al, 2002; Littlepage \& Ruderman, 2002)partially contains the MTS of the kinase, and it is lost upon AURKA import into the mitochondrial matrix (Bertolin et al, 2018; Grant et al, 2018). In this light, it is tempting to speculate that the mitochondrial import of AURKA is a way of protecting the kinase from proteasome-targeted ubiquitylation, allowing AURKA to perform selective functions on the IMM as the interaction with the mitophagy receptor PHB2. Future studies are mandatory to elucidate these first steps in AURKA-dependent mitophagy, together with the definition of the other molecular players involved.

Another intriguing observation is that AURKA acts on mitophagy without inducing the loss of mitochondrial biogenesis markers as MT-C01, SDHA, and PGC1 $\alpha$. Whereas PGC1 $\alpha$ is a nuclear transcription factor, MT-CO1, and SDHA have a mitochondrial localisation. This implies that MT-CO1 and SDHA should be targeted by mitophagy as any other IMM/matrix protein. Nevertheless, it is interesting to notice that they remain unaffected (SDHA) or even increased (MTCO1) when mitophagy is induced by AURKA overexpression. These results offer multiple interpretations. First, they could indicate that the organelle degradation in AURKA-overexpressing cells does not follow the same kinetics for all mitochondrial proteins. Second, they could also suggest that selected mitochondrial proteins such as biogenesis factors are spared from degradation, perhaps through an mitochondrial derived vesicles-based mechanism (Soubannier et al, 2012). Last, they could hint at the fact that

specific siRNA was validated by Western blotting; the abundance of PARK2/Parkin was normalised to that of the loading control, which was arbitrarily fixed at 1 and reported below the blot. (C) Representative immunoblot and quantification of the normalised abundance of PARK2-Parkin and of MT-CO2 in total lysates of HEK293 cells transfected as indicated. (D) Loss of PMPCB staining (threshold mask and corresponding quantification) in MCF7 cells co-transfected with PARK2/Parkin-GFP and a control- or an AURKA-specific siRNA and treated with $4 \mathrm{~h}$ CCCP where indicated. $A=$ mitochondrial area normalised against total cell area (\%). $n=10$ cells per condition from one representative experiment (of three). The efficiency of the AURKA-specific siRNAs was validated by Western blotting; the abundance of AURKA was normalised to that of the loading control, which was arbitrarily fixed at one, and reported below the blot. $n=3$ independent experiments. (A, B, C) Data extend from minimum to maximum (A, B) or represent mean \pm SEM (C). Scale bar: $10 \mu \mathrm{m}$. Loading control: TUBA1A. (A, B, C) ${ }^{\star *} P<0.01,{ }^{* * *} \mathrm{P}<0.001$ against the "Parkin + AURKA" (A), the "AURKA + sictrl" (B) or the "Empty vector" (C) conditions. NS, not significant.

Source data are available for this figure. 
A

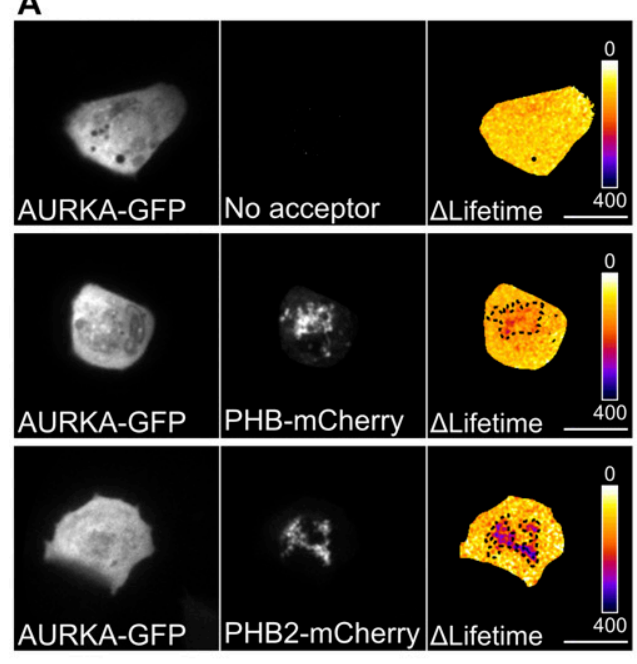

D

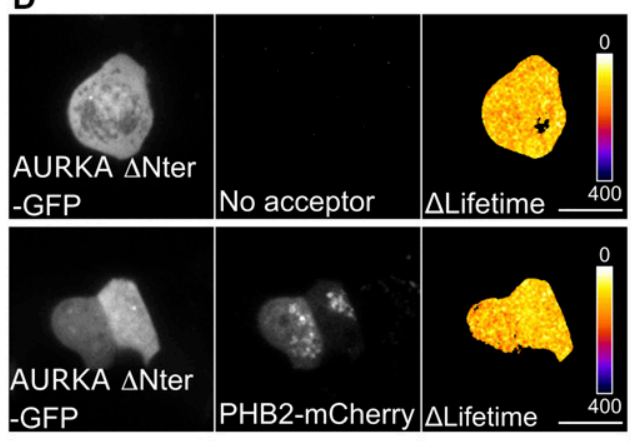

B

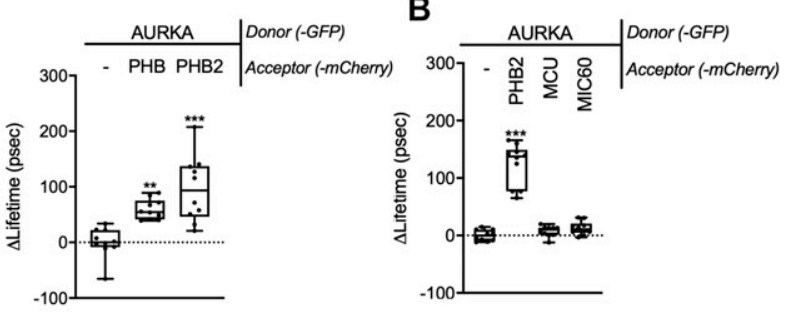

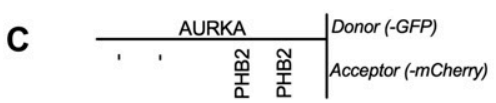
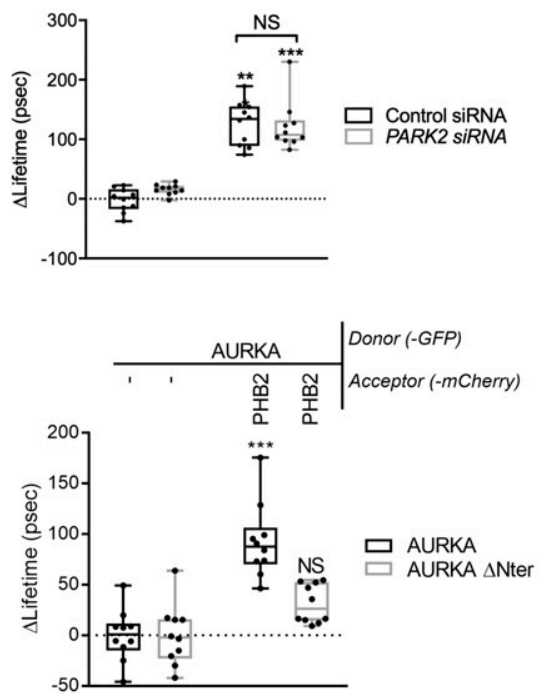
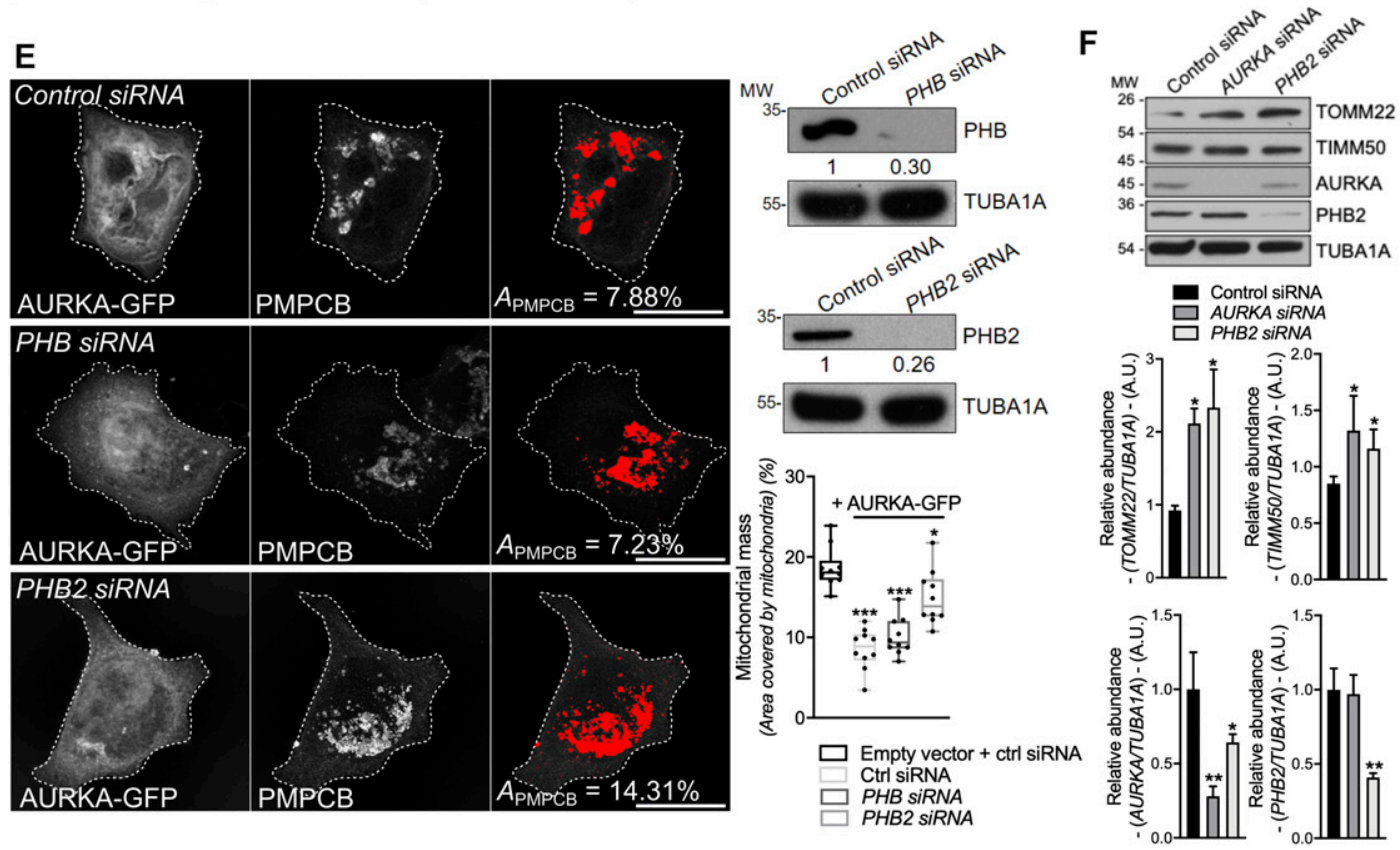

Figure 5. The interaction of AURKA with PHB2 is mandatory for mitophagy.

(A, B, C, D) Förster's Resonance Energy Transfer by Fluorescence Lifetime Imaging Microscopy analyses on MCF7 cells expressing AURKA-GFP alone or together with PHBmCherry or PHB2-mCherry (A), alone or together with PHB2-mCherry, MCU-mCherry, or MIC60-mCherry (B), co-expressing AURKA-GFP alone or together with PHB2mCherry in the presence of a control- or a PARK2-specific siRNA (C), or expressing AURKA $\triangle N$ ter-GFP alone or together with PHB2-mCherry (D). Pseudocolour scale: pixel-bypixel $\triangle$ Lifetime. Dots: mitochondria-rich area. (E) Loss of PMPCB staining (threshold mask and corresponding quantification) in MCF7 cells co-transfected with an empty vector or with AURKA-GFP and a control, a PHB-, or a PHB2-specific siRNA as indicated. A = mitochondrial area normalised against total cell area (\%). $n=10$ cells per 
mitochondrial biogenesis is constantly activated to induce a compensatory production of new organelles. This last option is corroborated by the fact that mitochondrial mass loss in AURKAoverexpressing cells is always partial, and mitochondria never entirely disappear as it occurs in other mitophagy paradigms (e.g., mitophagy induced within the PINK1/Parkin pathway). In this light, the partial loss of mitochondria could be seen as a balance between turnover and biogenesis mechanisms, where biogenesis continuously compensates organelle clearance. This possibility is also supported by complementary data obtained with mitoTandem, where GFP is never totally quenched into mitochondria upon AURKA overexpression. This would give a population of "new" mitochondria, and one of "old" organelles undergoing degradation. In terms of fluorescence, this would result in mitochondria with both GFP and mCherry ("new" mitochondria), and one with mCherry only ("old" mitochondria). Given that the mitochondrial population is mixed with "new" and "old" organelles, this explains why the mCherry fluorescence is higher than GFP when AURKA is overexpressed. Overall, these results open up the possibility that there is a tight regulation of the mitochondrial biogenesis/turnover ratio in cells overexpressing AURKA, and results obtained in HMLE cells strongly link the quantity of AURKA to its capacity of triggering mitophagy only, or mitophagy with concomitant biogenesis. This equilibrium appears to be optimised to maintain a population of metabolically competent organelles producing high quantities of ATP, and further studies are required to understand the molecular mechanisms of this regulation.

Given the breadth of the AURKA interactome, it is conceivable that the kinase plays different roles within the same subcellular compartment. However, a fascinating question-still unanswered-is whether one pool of AURKA (e.g., the mitochondrial one) is capable of triggering all the functions of the kinase at a given location, or whether sub-organellar pools of the protein exist. To optimise anticancer therapies, it is essential to explore how the kinase orchestrates its functions in time and space within one single subcellular compartment. Structural biology data revealed that the interaction of AURKA with a substrate needs a two-step conformational change. First, the kinase activates itself through the autophosphorylation on Thr288 (Walter et al, 2000; Cheetham, 2002; Bayliss et al, 2003; Zhang et al, 2007). This modification induces a "permissive" change in the conformation of the kinetic pocket, and increasing AURKA kinase activity in cells and in X. laevis (Cheetham, 2002; Littlepage et al, 2002; Zhang et al, 2007). Second, the interaction with an activator as TPX2 further buries the phosphorylated Thr288 in the kinetic pocket of the kinase, and makes it inaccessible to phosphatases (Bayliss et al, 2003). This locks the kinase in a fully active conformation, capable of interacting with its substrates (Kufer et al, 2002; Eyers et al, 2003; Brunet et al, 2004). Although the activation and activity events are needed for AURKA to phosphorylate its multiple partners (Nikonova et al, 2013), it should be noted that these events were mainly reported for mitotic interactors of AURKA at the mitotic spindle. Non-mitotic roles of AURKA are constantly arising (Tsunematsu et al, 2015; Korobeynikov et al, 2017; Bertolin \& Tramier, 2020); a still unanswered question is whether the activation/activity mechanisms during interphase and at specific subcellular locations are identical to the mitotic ones (Vaufrey et al, 2018). Most of the current AURKA inhibitors are designed to block the activation of the kinase by behaving as ATP analogues with higher affinity for the kinetic pocket (Damodaran et al, 2017). The efficacy of the available ATP analogues in anti-cancer therapies is poor, and none of the existing ones passed phase III of clinical trials (Bavetsias \& Linardopoulos, 2015). However, combinatorial approaches of AURKA inhibitors together with microtubule-stabilising drugs were shown to be beneficial to patients (Mazumdar et al, 2009; Lin et al, 2012; Sehdev et al, 2013). In addition, in silico (Kong et al, 2018) and high-content FRET/FLIM studies (Sizaire et al, 2020), which rely on AURKA conformational changes, represent exciting new strategies to identify novel AURKA inhibitors using the conformational changes of the kinase. In the context of the mitochondrial functions of AURKA, the future challenge will be to integrate ATP analogues with compounds targeting one specific conformation, one specific function of the kinase, and at a given subcellular location. That would selectively block only selected roles of AURKA, while leaving the others unaffected.

In the perspective of optimising combinatorial therapies in the future, it would be exciting to explore the effects of combining AURKA inhibitors and xanthohumol in vivo. This study represents the first piece of evidence that a single pharmacological compound can block at least two mitochondrial functions regulated by AURKA. Our data reinforce the concept that mitophagy and ATP production are in a mutual crosstalk (Melser et al, 2013, 2015), and they strongly suggest that overexpressed AURKA selects dysfunctional mitochondria for elimination, while sparing the metabolically efficient ones in a fused network (Bertolin et al, 2018). However, the selectivity of xanthohumol towards AURKA-dependent mitophagy should further be investigated. We are currently incapable of separating the roles of xanthohumol as a ligand of PHB2 (Yoshimaru et al, 2015) and as a Valosin-containing protein inhibitor in autophagy (Sasazawa et al, 2012). In the case of AURKA-dependent mitophagy, the addition of this compound does not alter the proximity of PHB2 with the other members of the tripartite complex, but rather the one of AURKA and MAP1LC3. This could be linked to the autophagy-blocking properties of xanthohumol playing a more prominent role in this experimental paradigm. In addition, FRET/FLIM data indicate that constituting a functional tripartite complex-where all protein-protein interactions occur-is necessary for mitophagy. In this light, the effect of xanthohumol and of the S39A variant could modify the structure of the tripartite complex, or even create two bi-partite sub-complexes. Overall, our data call for future studies to better understand how

condition from one representative experiment (of three). Scale bar: $10 \mu \mathrm{m}$. The efficiency of the PHB- and of the PHB2-specific siRNAs was validated by Western blotting; the abundance of PHB or of PHB2 was normalised to that of the loading control, which was arbitrarily fixed at 1, and reported below each blot. (F) Representative immunoblot and quantification of the normalised abundance of TOMM22, TIMM50, AURKA, and PHB2 in total lysates of T47D cells transfected with a control, AURKA-, or PHB2-specific siRNAs. Loading control: TUBA1A. $n=3$ independent experiments. (A, B, C, D, E, F) Data extend from min to max (A, B, C, D, E) or are means \pm SEM (F). (A, B, C, D, E, F) ${ }^{*} P<0.05,{ }^{* *} P<0.01,{ }^{* * *} P<0.001$ against each corresponding "AURKA-no acceptor" (A, B, C, D), the "Empty vector + Control siRNA" (E), or each "Control siRNA" (F) condition. NS, not significant.

Source data are available for this figure. 
A

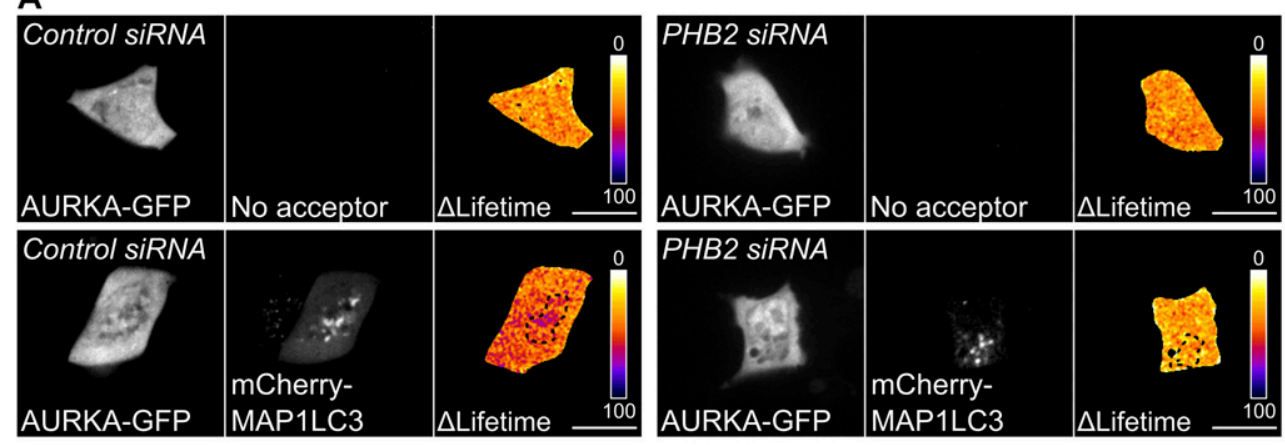

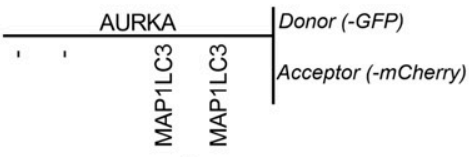

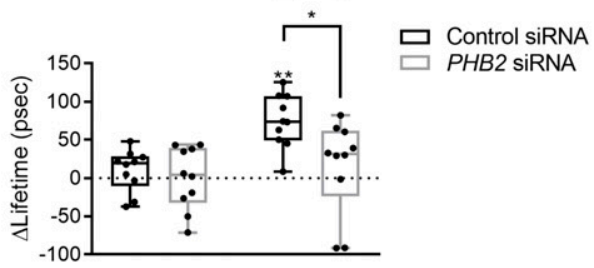

B SP1Q99623|PHB2_HUMAN SP $035129 \mid$ PHB2 ${ }^{-}$MOUSE SP|P50093|PHB2_CAEEL

-MAQNLKDLAGRLP-AGPRGMGTALKLLLGAGAVAYGVRESVFTVEGGHRAIFFNRIGGV 58 -MAQNLKDLAGRLP-AGPRGMGTALKLLLGAGAVAYGVRESVFTVEGGHRAIFFNRIGGV 58 KQGQEA--MKKAIQNARGAGVGLGLVAAAGAAVYGVAQSMFTVEAGHRAIMFNRIGGL 58 SP|A9UMS3|PHB2 2 XENTR -MAQNLKDFAGRLP-AGPRGMGTAMKLLLGAGAVAYAVKESVFTVEGGHRAIFFNRIGGV 58 TR|Q0E924 |Q0E920_DROME MAQSKLNDLAGKLGKGGPPGLGIGLKVLAAVGAAAYGVSQSLYTVEGGHRAIIFSRLGGI 60

C
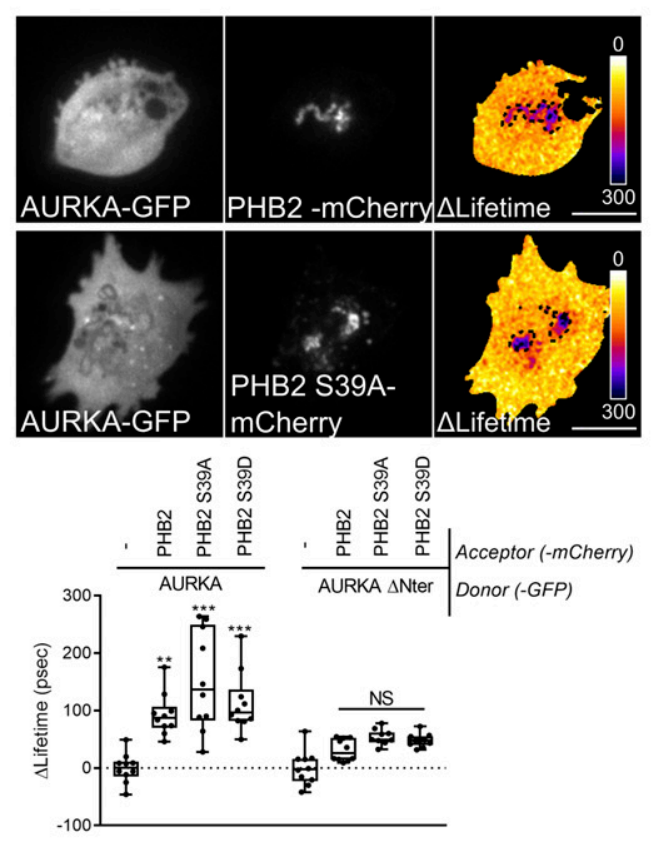

D
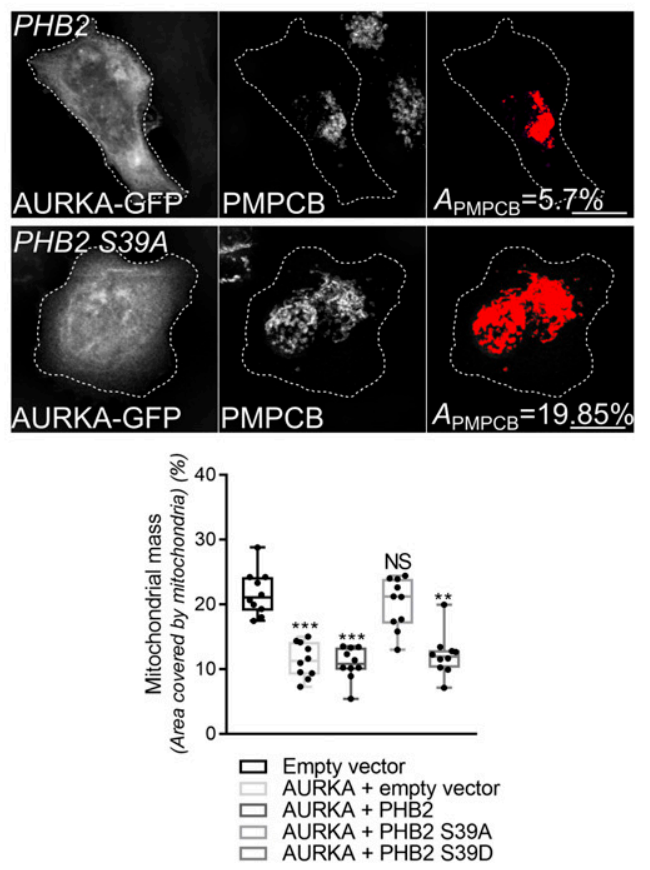

Figure 6. The phosphorylation of PHB2 on Ser39 is mandatory for mitophagy.

(A) Förster's Resonance Energy Transfer by Fluorescence Lifetime Imaging Microscopy analyses on MCF7 cells expressing AURKA-GFP alone or together with mCherryMAP1LC3B in the presence of a control or a PHB2-specific siRNA. Dotted area: autophagosome/autolysosome-rich areas. Pseudocolour scale: pixel-by-pixel $\Delta$ Lifetime. Graph: corresponding $\Delta$ Lifetime quantifications in the dotted area. $n=10$ cells per condition of one representative experiment (of three). (B) Clustal Omega multi-species alignment of the PHB2 region comprising Ser39. Ser39 is indicated in magenta. UniProt accession numbers used for the alignment are indicated. (C) Representative images and Förster's Resonance Energy Transfer by Fluorescence Lifetime Imaging Microscopy analyses on MCF7 cells expressing AURKA-GFP or AURKA $\triangle$ Nter-GFP alone, 
the tripartite complex is structurally organised, and how xanthohumol interferes with its stability.

In conclusion, we here show that overexpressed AURKA localised at mitochondria induces the degradation of mitochondria by mitophagy. This is achieved through the formation of a tripartite complex with MAP1LC3 and its IMM receptor PHB2. We also propose that this molecular mechanism is of relevance to cancer, as the remaining organelles form a fused mitochondrial network and show an increased capacity to produce ATP. Maintaining a population of highly competent mitochondria might represent a way to sustain the metabolic demands of the cancer cell, while eliminating the less efficient organelles. Finally, blocking mitophagy by pharmacological means translates in lowering ATP production rates, thereby paving the way to novel strategies in counteracting the effects of the overexpression of AURKA in epithelial cancers.

\section{Materials and Methods}

\section{Expression vectors and molecular cloning}

The list of plasmids used in this study is reported in Table S1. DNA constructs were either generated using the Gibson Assembly Master Mix (New England Biolabs), or the T4 DNA ligase (Thermo Fisher Scientific). Where indicated, plasmids were directly purchased from Addgene. Site-directed mutagenesis reactions were performed by Quick-Change site-directed mutagenesis (Stratagene). All cloning and mutagenesis reactions were verified on a 3130 XL sequencer (Applied Biosystems).

\section{Cell culture procedures}

MCF7 (HTB-22), T47D (HTB-133), and HEK293 (CRL-1573) cells were purchased from the American Type Culture Collection and were free from mycoplasma. They were grown in DMEM (Thermo Fisher Scientific) supplemented with 10\% FBS (Thermo Fisher Scientific), 1\% L-glutamine (Thermo Fisher Scientific) and $1 \%$ penicillinstreptomycin (Thermo Fisher Scientific). For all live microscopy experiments, cells were grown at $37^{\circ} \mathrm{C}$ in Nunc Lab-Tek II Chamber slides (Thermo Fisher Scientific). Standard growth media was replaced with phenol red-free Leibovitz's L-15 medium (Thermo Fisher Scientific) supplemented with $20 \%$ FBS and $1 \%$ penicillinstreptomycin before imaging. Mycoplasma-free HMLE cells were purchased from Lonza and they were grown in a mixture of $50 \%$ complete mammary epithelial growth medium (Lonza) and 50\% DMEM F12 (Thermo Fisher Scientific), supplemented with 10\% FBS, $0.01 \mathrm{mg} / \mathrm{ml}$ insulin (Sigma-Aldrich), and $0.48 \mu \mathrm{g} / \mathrm{ml}$ hydrocortisone (Sigma-Aldrich). For transfections, $1 \times 10^{7}$ cells $/ \mathrm{ml}$ were resuspended in $20 \mu \mathrm{l}$ of Buffer R and transfected according to the manufacturer's instructions. AllStars negative control (SI03650318) and functionally validated siRNAs against PHB (SI02223557) and PHB2 (SI02780918) were purchased from QIAGEN; the siRNA against AURKA was synthesised and purchased from Eurogentec, as previously described (Bertolin et al, 2016) (sequence: 5'-AUGCCCUGUCUUACUGUCA-3'). The PARK2-specific SiRNA was purchased from Thermo Fisher Scientific (HSS107593). The AURKA-specific shRNA (SHCLNG-NM_003600) and a non-targeting control (SHCO02) were purchased from Sigma-Aldrich. Plasmids and shRNAs were transfected by the calcium phosphate method or with Lipofectamine 2000 (Thermo Fisher Scientific), according to the manufacturer's instructions. Plasmids in HMLE cells were inserted by nucleofection with the Neon Transfection System (Thermo Fisher Scientific), by applying two pulses at 1,400 V for $20 \mathrm{~ms}$. SiRNAs were transfected using Lipofectamine RNAiMAX (Thermo Fisher Scientific); co-transfections of plasmids and siRNAs were made with Lipofectamine 2000 according to the manufacturer's instructions. Cells were plated at 70\% confluence in 24-well cell plates for immunocytochemistry, in eight-well or four-well Nunc LabTek II Chamber slides for live microscopy, or on $10 \mathrm{~cm}$ Petri dishes for total cell lysates or flow cytometry. Cells were harvested, fixed or imaged $48 \mathrm{~h}$ after transfection. For flow cytometry, cells were rinsed twice in $\mathrm{Ca}^{2+}$ - and $\mathrm{Mg}^{2+}$-free Phosphate Buffer Saline (Thermo Fisher Scientific), trypsinised (0.05\% Trypsin-EDTA, Thermo Fisher Scientific) and resuspended in phenol-free L-15 medium.

\section{Chemical reagents and fluorescent dyes}

Carbonyl cyanide 3-chlorophenylhydrazone (C2759; Sigma-Aldrich) was used at a final concentration of $10 \mu \mathrm{M}$ for $4 \mathrm{~h}$ before imaging. 3-methyladenine (S2767, 5 mM) and bafilomycin A1 (S1413, $100 \mathrm{nM}$ ) were purchased from Selleck Chemicals and incubated for $24 \mathrm{~h}$ before imaging or cell harvesting; MG-132 (S2619, $1 \mu \mathrm{M}$ ) was purchased from Selleck Chemicals and incubated for $16 \mathrm{~h}$; xanthohumol (S7889, $30 \mu \mathrm{M}$ [Sasazawa et al, 2012]) and MLN8237 (S1133, 50 nM) were purchased from Selleck Chemicals and incubated for $24 \mathrm{~h}$ before imaging or cell harvesting for Western blotting, or $6 \mathrm{~h}$ before OCR measurements. All compounds were resuspended in DMSO (UD8050A; Euromedex). Tetramethylrhodamine, methyl ester, perchlorate (TMRM, T668, 50 nM; Thermo Fisher Scientific), and MitoTracker Green FM (M7514, 100 nM; Thermo Fisher Scientific) were simultaneously added to phenol-free L-15 medium and incubated for 30 min at $37^{\circ} \mathrm{C}$ before imaging or flow cytometry. Lysotracker Red DND-99 (Thermo Fisher Scientific) was used at a final concentration of $50 \mathrm{nM}$ and incubated for $40 \mathrm{~min}$ at $37^{\circ} \mathrm{C}$ before flow cytometry.

\section{Drosophila strains}

D. melanogaster stocks and crossings were set up and grown at $25^{\circ} \mathrm{C}$. UAS-mito-HA-GFP (BDSC: 8443), UASp-mCherry-Atg8a (BDSC: 37750), and UAS-aurA.Exel (BDSC: 8377) were obtained from the Bloomington Drosophila Stock Center. The scabrous-GAL4 (sca-GAL4)

or together with normal PHB2, PHB2 S39A, or PHB2 S39D-mCherry. Graph: corresponding $\triangle$ Lifetime quantifications in the dotted area. Dots: mitochondria-rich area. Pseudocolour scale: pixel-by-pixel $\triangle$ Lifetime. (D) Loss of PMPCB staining (threshold mask and corresponding quantification) in MCF7 cells co-transfected with an empty vector or with AURKA-GFP and PHB2-6xHis, PHB2 S39A-6xHis, or PHB2 S39D-6xHis as indicated. $A=$ mitochondrial area normalised against total cell area (\%). $n=10$ cells per condition from one representative experiment (of three). Data extend from min to max. Scale bar: $10 \mu \mathrm{m} .(\mathrm{A}, \mathrm{C}, \mathrm{D}){ }^{\star} P<0.05,{ }^{* *} P<0.01,{ }^{* * *} P<0.001$ against the “AURKAno acceptor" (A, C), or the "Empty vector" (D) conditions. NS, not significant. 
A
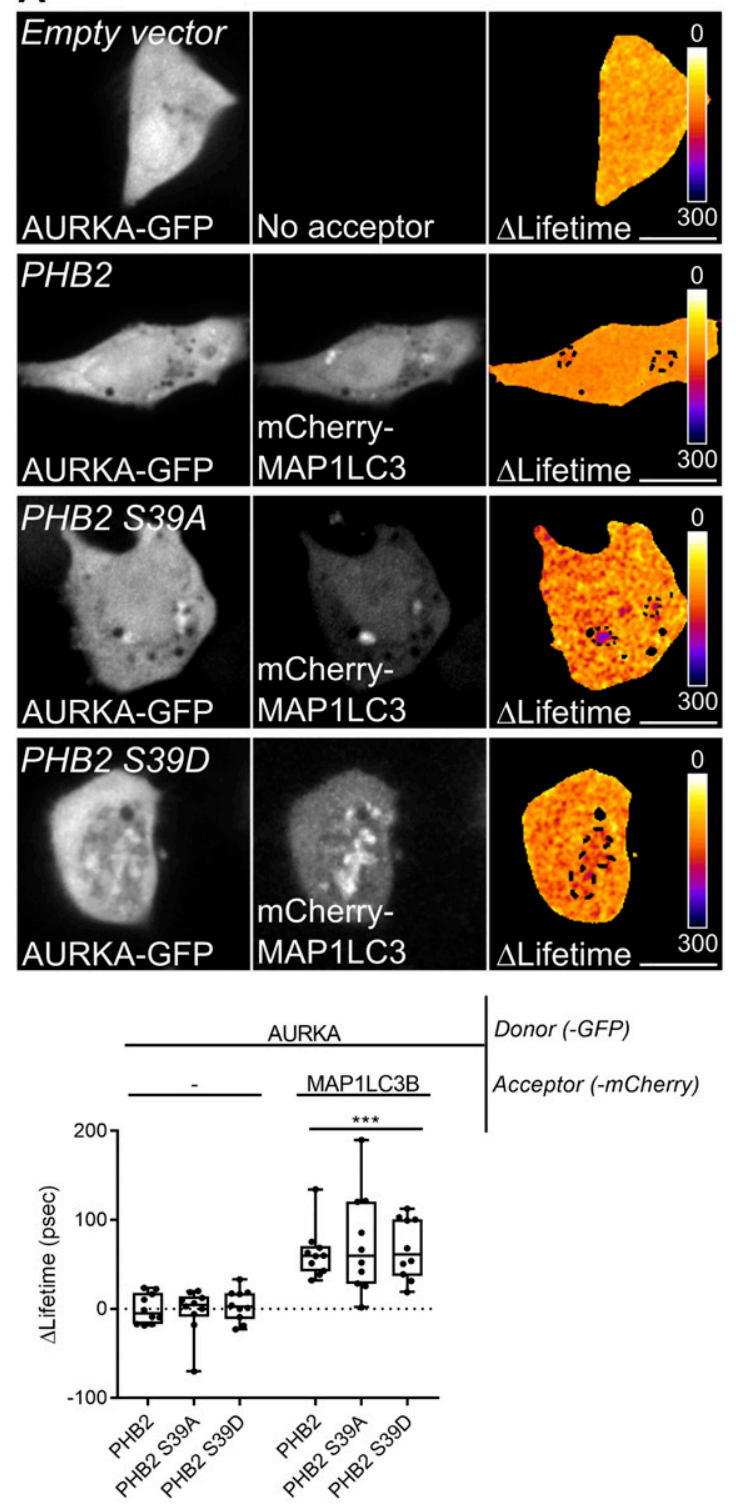

B
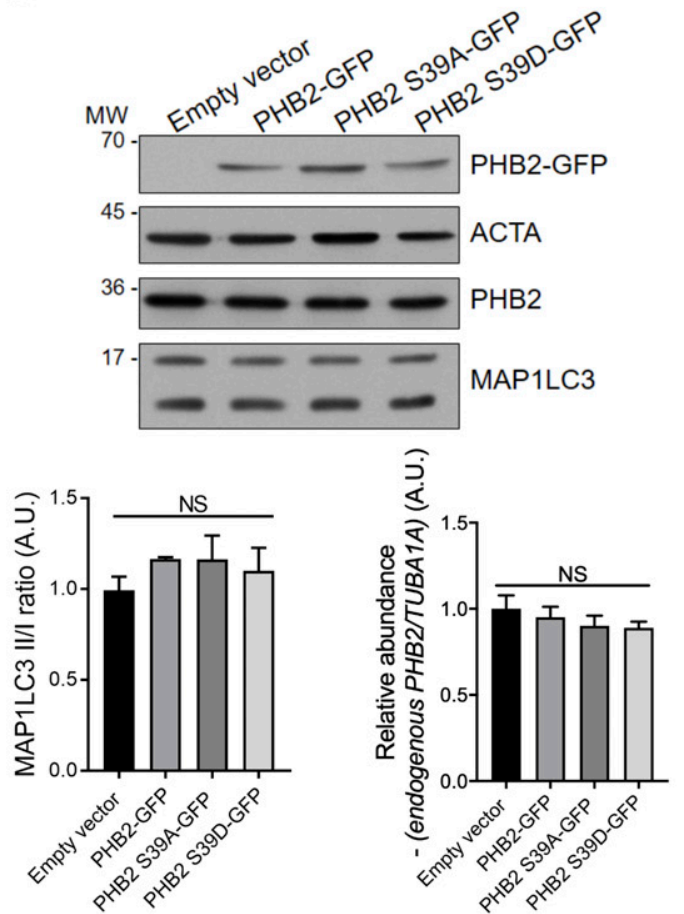

C
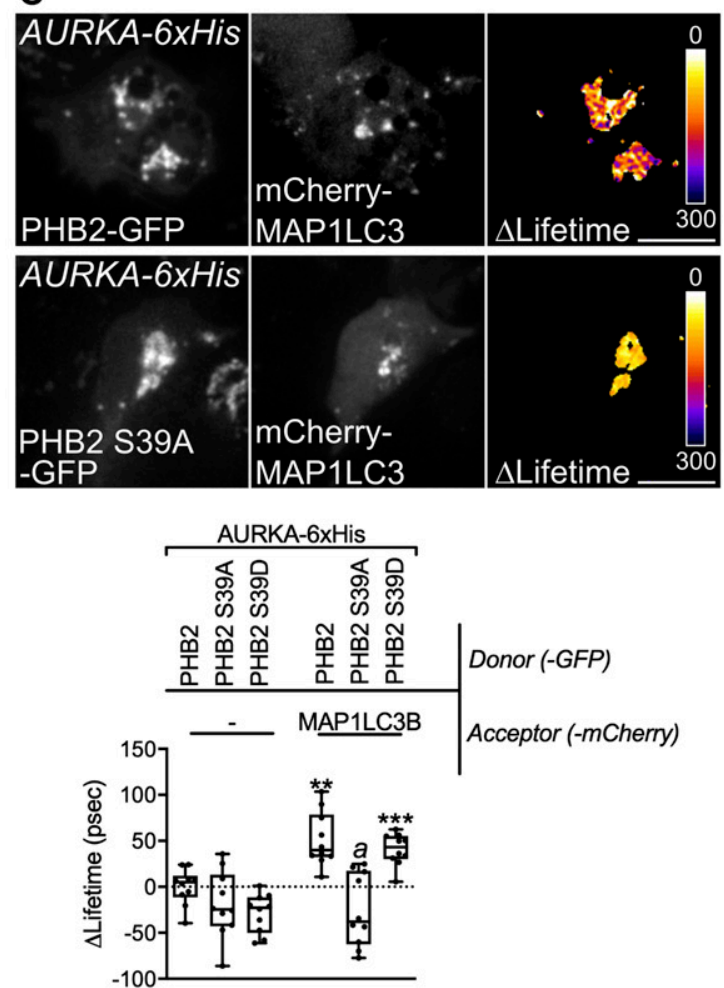

Figure 7. AURKA forms a tripartite complex with PHB2 and MAP1LC3 upon the induction of mitophagy.

(A) Förster's Resonance Energy Transfer by Fluorescence Lifetime Imaging Microscopy analyses on MCF7 cells expressing AURKA-GFP alone or together with mCherryMAP1LC3B, and in the presence of the indicated PHB2-6xHis variants. Dotted area: autophagosome/autolysosome-rich areas. Graph: corresponding $\triangle \mathrm{Lifetime}$ quantifications in the dotted area. $n=10$ cells per condition of one representative experiment (of three). (B) Representative immunoblot and quantification of the MAP1LC3II/I ratio and of the normalised abundance of endogenous PHB2 in total lysates of HEK293 cells transfected as indicated. Loading control: ACTA. $n=3$ independent experiments. (C) Förster's Resonance Energy Transfer by Fluorescence Lifetime Imaging Microscopy analyses on MCF7 cells co-expressing AURKA-6xHis and 
driver was obtained from Mlodzik et al (1990). aurA ${ }^{S T}$ and aurA ${ }^{2 G}$ were used as in Bertolin et al (2018). $w^{1118}$ pupae were used as wild-type controls for all experiments. All crossings and the corresponding abbreviations used in the article are listed in Table S2. Pupae were collected as white pupae, aged for $16 \mathrm{~h}$ at $25^{\circ} \mathrm{C}$ and mounted on glass slides before imaging. All images collected in this study were acquired from epithelial cells of the dorsal thorax (notum) at room temperature.

\section{Western blotting procedures}

Total protein fractions were obtained by lysing HEK cells in $50 \mathrm{mM}$ Tris- $\mathrm{HCl}$ (pH 7.5), $150 \mathrm{mM} \mathrm{NaCl}, 1.5 \mathrm{mM} \mathrm{MgCl} 2,1 \%$ Triton X-100, and $0.5 \mathrm{mM}$ DT, supplemented with $0.2 \mathrm{mM} \mathrm{Na}_{3} \mathrm{VO}_{4}, 4 \mathrm{mg} / \mathrm{ml} \mathrm{NaF}, 5.4 \mathrm{mg} / \mathrm{ml} \beta-$ glycerophosphate and protease inhibitors (Complete Cocktail; Roche). Lysates were centrifuged at $13,000 \mathrm{~g}$ for $20 \mathrm{~min}$ at $4^{\circ} \mathrm{C}$ and frozen at $-80^{\circ} \mathrm{C}$ for long-term storage. All protein fractions were quantified using the Bradford reagent (Bio-Rad). After resuspension in Laemmli sample buffer and boiling protein fractions were resolved by SDS-PAGE, transferred onto a nitrocellulose membrane (GE Healthcare) and analysed by Western blotting. The list of primary antibodies and their working dilutions are provided in Table S3. The home-made anti-MT-CO2 antibody was provided by Dr. Anne Lombès (Cochin Institute) and published previously (Agier et al, 2012). Anti-mouse and anti-rabbit secondary antibodies conjugated to the HRP were purchased from Jackson ImmunoResearch Laboratories; anti-rat secondary antibodies conjugated to HRP were purchased from Bethyl Laboratories. The membranes were incubated with commercially available developing kit (Pierce), or a homemade enhanced chemiluminescence substrate made of $100 \mathrm{mM}$ Tris ( $\mathrm{pH}$ 8.6), $13 \mathrm{mg} / \mathrm{ml}$ coumaric acid (Sigma-Aldrich), $44 \mathrm{mg} / \mathrm{ml}$ luminol (Sigma-Aldrich), and 3\% hydrogen peroxyde. Chemiluminescence signals were captured on film (Thermo Fisher Scientific), developed using a CURIX 60 developer (Agfa Healthcare) and quantified with Fiji software $(\mathrm{NIH})$. The relative abundance of specific bands of interest was calculated by normalising the integrated density of each band towards the integrated density of loading controls. The uncropped blots of the present study are integrated in Supplemental Data 1.

\section{Transmission electron microscopy}

Cells were rinsed with $0.15 \mathrm{M}$ sodium cacodylate and with $2.5 \%$ glutaraldehyde for $1 \mathrm{~h}$. After fixation, the cells were rinsed with $0.15 \mathrm{M}$ sodium cacodylate and post-fixed with 1.5\% osmium tetroxide for $1 \mathrm{~h}$. After further rinsing, the samples were dehydrated in increasing concentrations of ethanol $(50 \%, 70 \%, 90 \%$ and $100 \% \mathrm{vol} / \mathrm{vol})$. The cells were gradually infiltrated with increasing concentrations of epoxy resin (30\%, 50\%, 70\% $\mathrm{vol} / \mathrm{vol}$ in ethanol) for a minimum of $3 \mathrm{~h}$ per concentration. The samples were then incubated overnight in pure epoxy resin before continuing with a two-step incubation in 2,4,6-Tris(dimethylamino-methyl)phenol (DMP30;
Sigma-Aldrich)-epoxy resin, first for $3 \mathrm{~h}$ and then for $24 \mathrm{~h}$ at $60^{\circ} \mathrm{C}$ to polymerise the samples en bloc. Ultra-thin sections of $80 \mathrm{~nm}$ were cut from the blocks using a UC7 ultramicrotome (Leica), placed on grids, post-stained with uranyl acetate for $30 \mathrm{~min}$ and with lead citrate for $20 \mathrm{~min}$. Sections were examined using a JEM-1400 Electron Microscope (JEOL Ltd.) operating at $120 \mathrm{kV}$ accelerating voltage. Digital images were acquired using a Gatan SC1000 Orius CCD camera with a dedicated imaging software (GataDigitalMicrographTM).

\section{Immunocytochemistry, confocal and FLIM microscopy}

Cells were fixed in $4 \%$ paraformaldehyde (Euromedex), stained using standard immunocytochemical procedures, and mounted in ProLong Gold Antifade reagent (Thermo Fisher Scientific). The antibodies used were primary monoclonal mouse anti-TOMM22 (Ab10436; Abcam); polyclonal rabbit anti-PMPCB, 1:500 (16064-1-AP; Proteintech); and secondary antimouse or antirabbit antibodies conjugated to Alexa 674 at a 1:500 dilution and Alexa 555 or 488 both at a 1:5,000 dilution (Thermo Fisher Scientific). Multicolour images of cultured cells were acquired with a Leica SP8 inverted confocal microscope (Leica) and a $63 \times$ oil-immersion objective (NA 1.4), a Leica SP5 inverted confocal microscope (Leica), and a $63 \times$ oil-immersion objective (NA 1.4) both driven by the Leica Acquisition Suite software, or alternatively with a BX61WI FV-1000 confocal microscope (Olympus) driven by Olympus FV-1000 software and equipped with a $60 \times$ oil-immersion objective (NA 1.35). The excitation and emission wavelengths for GFP/MitoTracker Green were 488 and 525/50 nm, respectively; for TMRM/mCherry/Alexa 555, they were 561 and 605/70 nm; for Alexa 647 they were 633 and 650/20 nm. GFP was used as a FRET donor in all experiments, its decrease was measured by FLIM microscopy and the corresponding $\Delta$ Lifetime was calculated using the Inscoper Suite (Inscoper) as in Bertolin et al (2019). Fluorescence colocalisation was calculated with the JaCoP plugin (Bolte \& Cordelieres, 2006) of the Fiji software after applying an automatic threshold mask to the confocal images. The same plugin was used to calculate TOMM22-negative/PMPCB-positive objects by normalising the objects with both mitochondrial markers on the total number of PMPCB-positive objects per image. This yielded values between 0 and 1, and the PMPCB-only objects were calculated by subtracting each of the TOMM22-positive/PMPCB-positive values from 1. The relative mitochondrial area $(A)$ was used to express mitochondrial mass, and it was calculated with Fiji software on maximal projections of confocal images acquired as above and following a previously validated procedure (Bertolin et al, 2013). It was represented as the ratio between the area covered by the mitochondrial stain (TOMM22 or PMPCB), selected with an automatic threshold mask, and the total cell area.

\section{Flow cytometry}

After incubation with MitoTracker Green FM or LysoTracker Red DND-99, cells were rinsed with PBS, trypsinised, and centrifuged at

PHB2-GFP or its S39A and S39D variants, alone or together with mCherry-MAP1LC3B. Graph: corresponding $\Delta$ Lifetime quantifications. $n=10$ cells per condition of one representative experiment (of three). Pseudocolour scale: pixel-by-pixel $\Delta$ Lifetime. Scale bar: $10 \mu \mathrm{m}$. (A, C) Data represent means \pm SEM, unless in (A, C) where they extend from min to max. (A, C) ${ }^{* * P}<0.01,{ }^{* *} P<0.001$ compared with each corresponding "No acceptor" $(\mathrm{A}, \mathrm{C})$ conditions in cells transfected with AURKA and PHB2. ${ }^{a} P<0.001$ compared with the "PHB2-MAP1LC3" or the "PHB2 S39A-MAP1LC3" conditions. NS, not significant; A.U., arbitrary units. Source data are available for this figure. 
A

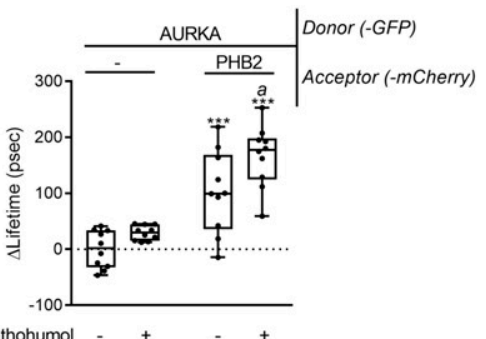

Xanthohumo

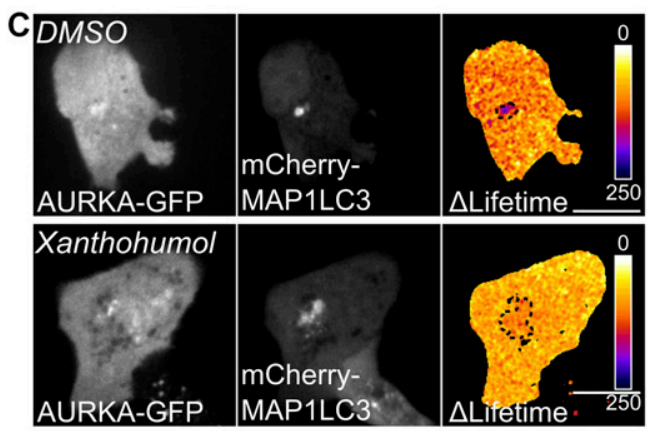

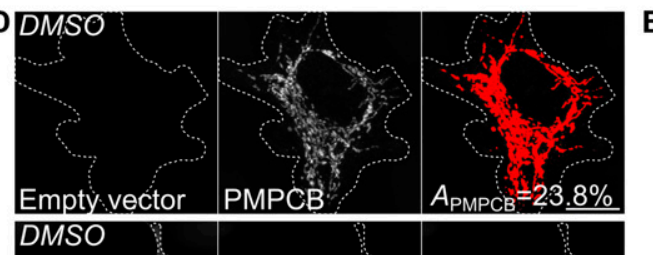
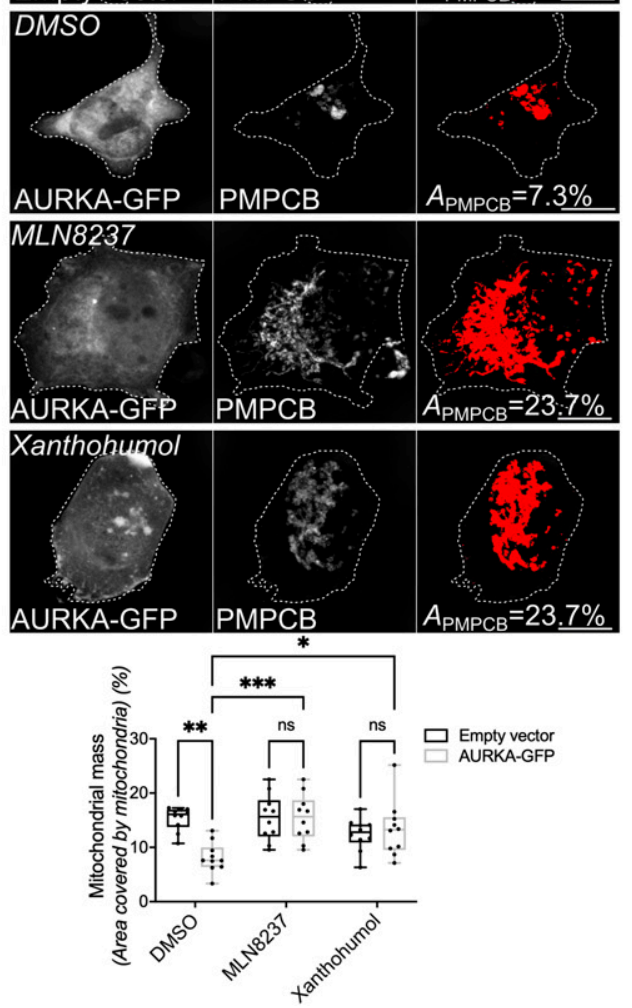

B

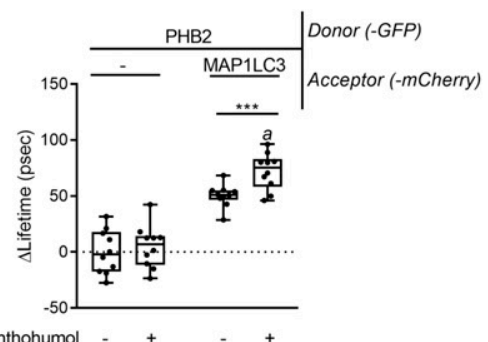

Xanthohum

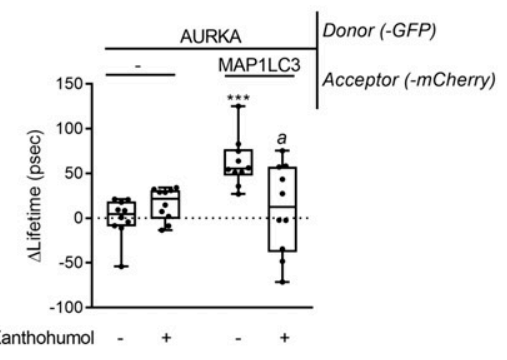

E

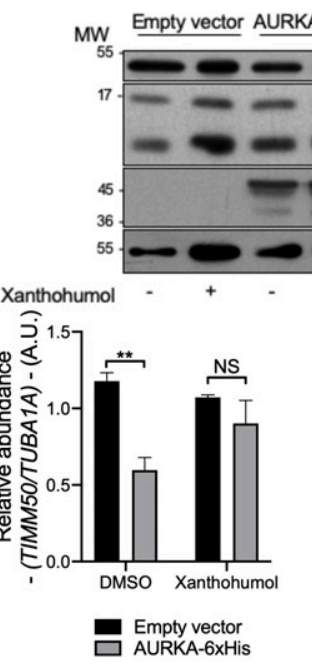

F $\frac{\widehat{D}}{\overline{0}}$

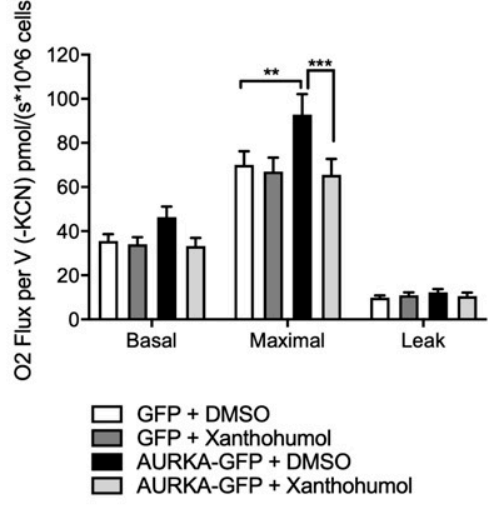

Figure 8. Treatment with xanthohumol blocks the interaction of AURKA with MAP1LC3, impairs mitophagy, and blocks AURKA-dependent ATP increase.

(A, B, C) Förster's Resonance Energy

Transfer by Fluorescence Lifetime Imaging Microscopy analyses on MCF7 cells expressing AURKA-GFP alone or together with PHB2-mCherry (A), PHB2-GFP alone or together with mCherry-MAP1LC3B (B), or AURKA-GFP alone or together with mCherry-MAP1LC3B (C) and treated with DMSO or with xanthohumol for $24 \mathrm{~h}$. Dotted area: autophagosome/autolysosomerich areas. $n=10$ cells per condition of one representative experiment (of three).

(D) Loss of PMPCB staining (threshold mask and corresponding quantification) in MCF7 cells co-transfected with AURKA-GFP and treated as indicated. $A=$

mitochondrial area normalised against total cell area (\%). $n=10$ cells per condition from one representative experiment (of three). (E) Representative immunoblot and quantification of the normalised abundance of TIMM50 and the MAP1LC3II/I ratio in total lysates of HEK293 cells transfected as indicated and treated with DMSO or xanthohumol for $24 \mathrm{~h}$. Loading control: TUBA1A. $n=3$ independent experiments. (F) Mitochondrial respiration of HEK293 cells overexpressing GFP or AURKA-GFP and treated with DMSO or xanthohumol. $n=3$ independent experiments. Pseudocolour scale: pixel-by-pixel $\Delta$ Lifetime. Scale bar: $10 \mu \mathrm{m}$. (E) Data extend from the $10^{\text {th }}$ to the $90^{\text {th }}$ percentile unless in $(E)$, where they represent means \pm SEM. (A, B, C, D, E, F $){ }^{*} P$ $<0.05,{ }^{* *} P<0.01,{ }^{* * *} P<0.001$ compared with each corresponding "No acceptor" (A, B, C), "AURKA-GFP DMSO" (D), "DMSO" (E), or "GFP + DMSO" or "AURKA-GFP + DMSO" (F) conditions. (A, B, C) $a={ }^{* *} P<0.01$ compared to each corresponding "-" condition (A, B, C) NS, not significant; A.U., arbitrary units. Source data are available for this figure. 


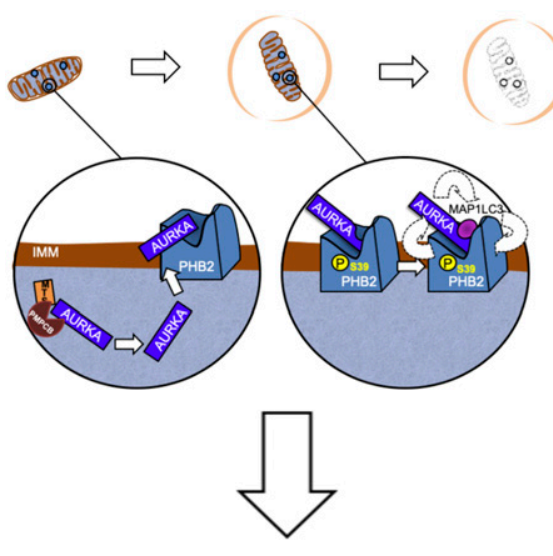

High ATP levels
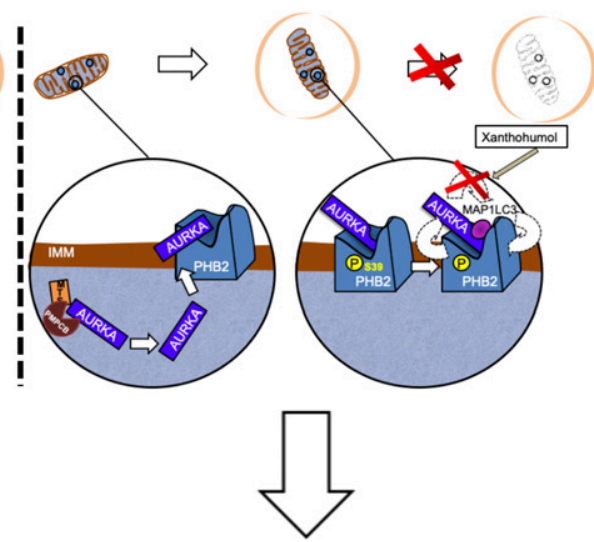

Normal ATP levels
Figure 9. Formation of the tripartite AURKA-PHB2-MAP1LC3 complex during AURKAdependent mitophagy, and potential inhibition strategies with xanthohumol.

Left panels: after being imported into mitochondria, AURKA interacts with the mitophagy receptor PHB2. The phosphorylation of Ser39 on PHB2 is mandatory for a functional AURKA-PHB2-MAP1LC3 complex to form and to complete mitochondrial clearance in paradigms when AURKA is overexpressed. Under these conditions, mitochondrial ATP levels are high. Protein-protein interactions within the tripartite complex are illustrated with dashed arrows. Right panels: a functional tripartite complex can be blocked by using the PHB2 ligand xanthohumol. This compound alters AURKA-MAP1LC3 proximity, creating a dysfunctional tripartite complex. This results in impaired mitophagy and in restoration of normal ATP levels.
$800 \mathrm{~g}$. Pellets were resuspended in sterile PBS, and the relative fluorescence intensity was measured with a FC500 flow cytometer (Beckman Coulter)

\section{OCR measurements}

Before OCR measurements, the cells were trypsinised and resuspended in normal growth medium. Measurements were carried out in the respiratory chamber of an Oroboros Oxygraph-2k (WGT). Cellular respiration was determined under basal conditions, with oligomycin (1 mg/ml; Sigma-Aldrich) to estimate leakage, and in the presence of increasing amounts (2.5-5 mM) of CCCP (Sigma-Aldrich) to calculate maximal respiration. To inhibit mitochondrial respiration, $1 \mathrm{mM}$ potassium cyanide was added (KCN; Sigma-Aldrich).

\section{Statistical analyses}

Two-way ANOVA tests were used to compare two variables among multiple conditions and one-way ANOVA tests were used to test one variable among multiple conditions, and $t$ test or the MannWhitney test were used to compare two conditions. All tests were performed after testing data for normality.

Two-way ANOVA and the Holm-Sidak method were used to compare the effect of the transfection conditions and the quantity of DNA nucleofected in HMLE cells (Fig S2), the effect of the transfection conditions and of the status of the autophagy flux on the number of MAP1LC3 dots per cell (Fig S3B), the pharmacological treatment and the fluorescent proteins on $\triangle$ Lifetime measurements (Figs 8A-C, S4B, and S5C), the effect of the siRNA downregulation strategy and the fluorescent protein on $\Delta$ Lifetime measurements (Figs $5 B$ and $C$ and $6 A$ and $B$ ), the effect of the the siRNA down-regulation strategy and plasmid expression on the relative mitochondrial area (Fig 5D), the effect of PHB2 isoforms and the fluorescent proteins on $\triangle$ Lifetime measurements (Fig 7A and C), the effect of transfection conditions and pharmacological treatments on the relative mitochondrial area (Fig 8D), the effect of the pharmacological treatment and the transfected vectors on the MAP1LC3II/I cleavage ratio and on the abundance of mitochondrial markers (Fig 8E), and the effect of the pharmacological treatment and the mitochondrial respiratory parameter on mitochondrial respiration (Fig 8F).

One-way ANOVA and the Holm-Sidak method were used to compare mitochondrial mass calculated as the relative mitochondrial area (Fig 1A), the MAP1LC3II/I cleavage ratio (Fig 1B), the effect of the Drosophila genotype on the quantity of Atg8 at mitochondria (Fig S1D), the abundance of lysosomes in electron microscopy experiments (Fig 2A), relative LysoTracker Red intensities (Fig 2B), the red/green fluorescence ratio in cells transfected with mitoTandem (Fig S3A), and pharmacological treatments on the relative mitochondrial area (Fig $3 \mathrm{~A}$ and C). One-way ANOVA on ranks and the Kruskal-Wallis method were used to compare relative MitoTracker Green intensities (Fig 1C), the abundance of mitochondrial markers (Figs $1 \mathrm{~F}$ and $7 \mathrm{~B}$ ), the MAP1LC3II/ I cleavage ratio (Fig 7B), $\triangle$ Lifetime measurements with components of the autophagy pathway (Fig 2C) or within the PHB complex (Fig 5A), and the relative mitochondrial area (Figs 4D and 6D).

$t$ test was used to compare the relative abundance of mitochondrial markers (Figs $1 \mathrm{~B}$ and $4 \mathrm{~B}$ and $\mathrm{C}$ ), the relative intensity of TMRM (Fig S1C) $\Delta$ Lifetime measurements (Fig 2D), pharmacological treatments on the relative mitochondrial area (Fig S4A and B), Mander's colocalisation coefficients (Figs 3D and 4A). The Mann-Whitney test was used to compare Mander's colocalisation coefficients (Fig 1C), the relative abundance of AURKA and of AURKA $\triangle N$ ter (Fig S1A), the effect of control or AURKA-specific siRNAs (Fig S1B), and the relative abundance of mitochondrial markers (Fig 4C).

The replicates for three independent experiments were shown for key quantifications in the manuscript, and illustrations were made using the SuperPlotsofData software (https:/ / huygens.science.uva.nl/ SuperPlotsOfData/) (Goedhart, 2021).

\section{Supplementary Information}

Supplementary Information is available at https://doi.org/10.26508/lsa. 202000806 


\section{Acknowledgements}

We thank Drs. L Buhlman and E Baldini for critical reading and helpful discussions. We also thank Dr. A Lombès for the generous gift of the anti-MT-CO2 primary antibody, Dr. S Jakobs for the gift of MIC60 CDNA, and Dr. B Levine for the gift of PHB and PHB2 CDNAs. We thank $L$ Deleurme of the flow cytometry and cell sorting platform (Biologie, Santé, Innovation Technologique, BIOSIT, Rennes, France), and we are grateful to S Ley-Ngardigal and EB Gökerküçük for critical reading and technical assistance. We also thank $S$ Dutertre and X Pinson from the Microscopy-Rennes Imaging Center (MRic, BIOSIT, Rennes, France) for advice, critical reading, and constructive comments on image analysis. MRic is member of the national infrastructure France-Biolmaging supported by the French $\mathrm{Na}-$ tional Research Agency (ANR-10-INBS-04). This work was supported by the Centre National de la Recherche Scientifique, the Ligue Contre le Cancer Comités d'llle et Vilaine, des Côtes d'Armor et du Finistère, and the Association pour la Recherche Contre le Cancer (ARC) to G Bertolin.

\section{Author Contributions}

G Bertolin: conceptualization, resources, data curation, formal analysis, funding acquisition, validation, investigation, visualization, methodology, project administration, and writing-original draft, review, and editing.

M-C Alves-Guerra: formal analysis, methodology, and writingreview and editing.

A Cheron: formal analysis, investigation, and methodology.

A Burel: formal analysis, investigation, visualization, methodology, and writing-review and editing.

C Prigent: resources, validation, and writing-review and editing. $\mathrm{R}$ Le Borgne: resources, validation, investigation, methodology, and writing-review and editing.

M Tramier: resources, validation, methodology, and writing-review and editing.

\section{Conflict of Interest Statement}

The authors declare that they have no conflict of interest.

\section{References}

Agier V, Oliviero P, Lainé J, L'Hermitte-Stead C, Girard S, Fillaut S, Jardel C, Bouillaud F, Bulteau AL, Lombès A (2012) Defective mitochondrial fusion, altered respiratory function, and distorted cristae structure in skin fibroblasts with heterozygous OPA1 mutations. Biochim Biophys Acta 1822: 1570-1580. doi:10.1016/j.bbadis.2012.07.002

Andrésson T, Ruderman JV (1998) The kinase Eg2 is a component of the Xenopus oocyte progesterone-activated signaling pathway. EMBO 17 : 5627-5637. doi:10.1093/emboj/17.19.5627

Bavetsias V, Linardopoulos S (2015) Aurora kinase inhibitors: Current status and outlook. Front Oncol 5: 278. doi:10.3389/fonc.2015.00278

Bayliss R, Sardon T, Vernos I, Conti E (2003) Structural basis of Aurora-A activation by TPX2 at the mitotic spindle. Mol Cell 12: 851-862. doi:10.1016/s1097-2765(03)00392-7

Bertolin G, Ferrando-Miguel R, Jacoupy M, Traver S, Grenier K, Greene AW, Dauphin A, Waharte F, Bayot A, Salamero J, et al (2013) The TOMM machinery is a molecular switch in PINK1 and PARK2/PARKINdependent mitochondrial clearance. Autophagy 9: 1801-1817. doi:10.4161/auto.25884
Bertolin G, Sizaire F, Herbomel G, Reboutier D, Prigent C, Tramier M (2016) A FRET biosensor reveals spatiotemporal activation and functions of aurora kinase A in living cells. Nat Commun 7: 12674. doi:10.1038/ ncomms12674

Bertolin G, Bulteau A-L, Alves-Guerra M-C, Burel A, Lavault M-T, Gavard O, Le Bras S, Gagné J-P, Poirier GG, Le Borgne R, et al (2018) Aurora kinase A localises to mitochondria to control organelle dynamics and energy production. Elife 7: e38111. doi:10.7554/elife.38111

Bertolin G, Sizaire F, Déméautis C, Chapuis C, Mérola F, Erard M, Tramier M (2019) Optimized FRET pairs and quantification approaches to detect the activation of aurora kinase A at mitosis. ACS Sens 4: 2018-2027. doi:10.1021/acssensors.9b00486

Bertolin G, Tramier M (2020) Insights into the non-mitotic functions of aurora kinase A: More than just cell division. Cell Mol Life Sci 77: 1031-1047. doi:10.1007/s00018-019-03310-2

Bolte S, Cordelieres FP (2006) A guided tour into subcellular colocalization analysis in light microscopy. J Microsc 224: 213-232. doi:10.1111/j.13652818.2006.01706.x

Brunet S, Sardon T, Zimmerman T, Wittmann T, Pepperkok R, Karsenti E, Vernos I (2004) Characterization of the TPX2 domains involved in microtubule nucleation and spindle assembly in Xenopus egg extracts. Mol Biol Cell 15: 5318-5328. doi:10.1091/mbc.e04-05-0385

Castro A, Vigneron S, Bernis C, Labbé J-C, Prigent C, Lorca T (2002) The D-Boxactivating domain (DAD) is a new proteolysis signal that stimulates the silent D-Box sequence of Aurora-A. EMBO Rep 3: 1209-1214. doi:10.1093/embo-reports/kvf241

Chan NC, Salazar AM, Pham AH, Sweredoski MJ, Kolawa NJ, Graham RLJ, Hess S, Chan DC (2011) Broad activation of the ubiquitin-proteasome system by Parkin is critical for mitophagy. Hum Mol Genet 20: 1726-1737. doi:10.1093/hmg/ddr048

Cheetham GMT (2002) Crystal structure of aurora-2, an oncogenic serine/ threonine kinase. J Biol Chem 277: 42419-42422. doi:10.1074/ jbc.c200426200

Chen BB, Glasser JR, Coon TA, Mallampalli RK (2013) Skp-cullin-F box E3 ligase component FBXL2 ubiquitinates Aurora B to inhibit tumorigenesis. Cell Death Dis 4: e759. doi:10.1038/cddis.2013.271

Coon TA, Glasser JR, Mallampalli RK, Chen BB (2012) Novel E3 ligase component FBXL7 ubiquitinates and degrades Aurora A, causing mitotic arrest. Cell Cycle 11: 721-729. doi:10.4161/cc.11.4.19171

Damodaran AP, Vaufrey L, Gavard O, Prigent C (2017) Aurora A kinase is a priority pharmaceutical target for the treatment of cancers. Trends Pharmacol Sci 38: 687-700. doi:10.1016/j.tips.2017.05.003

Di Rita A, Peschiaroli A, D'Acunzo P, Strobbe D, Hu Z, Gruber J, Nygaard M, Lambrughi M, Melino G, Papaleo E, et al (2018) HUWE1 E3 ligase promotes PINK1/PARKIN-independent mitophagy by regulating AMBRA1 activation via IKKa. Nat Commun 9: 3755. doi:10.1038/s41467018-05722-3

Eyers PA, Erikson E, Chen LG, Maller JL (2003) A novel mechanism for activation of the protein kinase Aurora A. Curr Biol 13: 691-697. doi:10.1016/s0960-9822(03)00166-0

Farag SS (2011) The potential role of aurora kinase inhibitors in haematological malignancies: Review. Br J Haematol 155: 561-579. doi:10.1111/j.1365-2141.2011.08898.x

Fujii Y, Yada M, Nishiyama M, Kamura T, Takahashi H, Tsunematsu R, Susaki E, Nakagawa T, Matsumoto A, Nakayama KI (2006) Fbxw7 contributes to tumor suppression by targeting multiple proteins for ubiquitindependent degradation. Cancer Sci 97: 729-736. doi:10.1111/j.13497006.2006.00239.x

Geisler S, Holmström KM, Skujat D, Fiesel FC, Rothfuss OC, Kahle PJ, Springer W (2010) PINK1/Parkin-mediated mitophagy is dependent on VDAC1 and p62/SQSTM1. Nat Cell Biol 12: 119-131. doi:10.1038/ncb2012 
Giet R, McLean D, Descamps S, Lee MJ, Raff JW, Prigent C, Glover DM (2002) Drosophila Aurora A kinase is required to localize D-TACC to centrosomes and to regulate astral microtubules. I Cell Biol 156: 437-451. doi:10.1083/jcb.200108135

Glover DM, Leibowitz MH, McLean DA, Parry H (1995) Mutations in aurora prevent centrosome separation leading to the formation of monopolar spindles. Cell 81: 95-105. doi:10.1016/0092-8674(95)90374-7

Goedhart I (2021) SuperPlotsOfData: A web app for the transparent display and quantitative comparison of continuous data from different conditions. Mol Biol Cell 32: 470-474. doi:10.1091/mbc.E20-09-0583

Grant R, Abdelbaki A, Bertoldi A, Gavilan MP, Mansfeld J, Glover DM, Lindon C (2018) Constitutive regulation of mitochondrial morphology by Aurora A kinase depends on a predicted cryptic targeting sequence at the $\mathrm{N}$ terminus. Open Biol 8: 170272. doi:10.1098/rsob.170272

Hagedorn M, Delugin M, Abraldes I, Allain N, Belaud-Rotureau M-A, Turmo M, Prigent C, Loiseau H, Bikfalvi A, Javerzat S (2007) FBXW7/hCDC4 controls glioma cell proliferation in vitro and is a prognostic marker for survival in glioblastoma patients. Cell Div 2: 9. doi:10.1186/1747-1028-2-9

Hurley JH, Young LN (2017) Mechanisms of autophagy initiation. Annu Rev Biochem 86: 225-244. doi:10.1146/annurev-biochem-061516-044820

Isakson P, Lystad AH, Breen K, Koster G, Stenmark H, Simonsen A (2013) TRAF6 mediates ubiquitination of KIF23/MKLP1 and is required for midbody ring degradation by selective autophagy. Autophagy 9: 1955-1964. doi:10.4161/auto.26085

Kashatus DF, Lim K-H, Brady DC, Pershing NLK, Cox AD, Counter CM (2011) RALA and RALBP1 regulate mitochondrial fission at mitosis. Nat Cell Biol 13: 1108-1115. doi:10.1038/ncb2310

Kim I, Rodriguez-Enriquez S, Lemasters JJ (2007) Selective degradation of mitochondria by mitophagy. Arch Biochem Biophys 462: 245-253. doi:10.1016/j.abb.2007.03.034

Kinzel D, Boldt K, Davis EE, Burtscher I, Trümbach D, Diplas B, Attié-Bitach T, Wurst W, Katsanis N, Ueffing M, et al (2010) Pitchfork regulates primary cilia disassembly and left-right asymmetry. Dev Cell 19: 66-77. doi:10.1016/j.devcel.2010.06.005

Klionsky DJ, Abdelmohsen K, Abe A, Abedin MJ, Abeliovich H, Acevedo Arozena A, Adachi H, Adams CM, Adams PD, Adeli K, et al (2016) Guidelines for the use and interpretation of assays for monitoring autophagy (3rd edition). Autophagy 12: 1-222. doi:10.1080/15548627.2016.1139264

Kong Y, Bender A, Yan A (2018) Identification of novel aurora kinase A (AURKA) inhibitors via hierarchical ligand-based virtual screening. I Chem Inf Model 58: 36-47. doi:10.1021/acs.jcim.7b00300

Korobeynikov V, Deneka AY, Golemis EA (2017) Mechanisms for nonmitotic activation of Aurora-A at cilia. Biochem Soc Trans 45: 37-49. doi:10.1042/bst20160142

Kufer TA, Silljé HHW, Körner R, Gruss OJ, Meraldi P, Nigg EA (2002) Human TPX2 is required for targeting Aurora-A kinase to the spindle. J Cell Biol 158: 617-623. doi:10.1083/jcb.200204155

Lazarou M, Sliter DA, Kane LA, Sarraf SA, Wang C, Burman JL, Sideris DP, Fogel Al, Youle RJ (2015) The ubiquitin kinase PINK1 recruits autophagy receptors to induce mitophagy. Nature 524: 309-314. doi:10.1038/nature14893

Lee C-Y, Andersen RO, Cabernard C, Manning L, Tran KD, Lanskey MJ, Bashirullah A, Doe CQ (2006) Drosophila Aurora-A kinase inhibits neuroblast selfrenewal by regulating aPKC/Numb cortical polarity and spindle orientation. Genes Dev 20: 3464-3474. doi:10.1101/gad.1489406

Lee KH, Johmura Y, Yu L-R, Park J-E, Gao Y, Bang JK, Zhou M, Veenstra TD, Yeon Kim B, Lee KS (2012) Identification of a novel Wnt5a-CK1E-Dvl2-Plk1-mediated primary cilia disassembly pathway: Primary cilia disassembly by the Dvl2Plk1 complex. EMBO J 31: 3104-3117. doi:10.1038/emboj.2012.144

Leray A, Padilla-Parra S, Roul J, Héliot L, Tramier M (2013) Spatio-temporal quantification of FRET in living cells by fast time-domain FLIM: A comparative study of non-fitting methods [corrected]. PLoS One 8: e69335. doi:10.1371/journal.pone.0069335
Lin Y, Richards FM, Krippendorff B-F, Bramhall JL, Harrington JA, Bapiro TE, Robertson A, Zheleva D, Jodrell DI (2012) Paclitaxel and CYC3, an aurora kinase A inhibitor, synergise in pancreatic cancer cells but not bone marrow precursor cells. Br J Cancer 107: 1692-1701. doi:10.1038/bjc.2012.450

Lindon C, Grant R, Min M (2016) Ubiquitin-mediated degradation of aurora kinases. Front Oncol 5: 307. doi:10.3389/fonc.2015.00307

Littlepage LE, Ruderman JV (2002) Identification of a new APC/C recognition domain, the A box, which is required for the Cdh1-dependent destruction of the kinase Aurora-A during mitotic exit. Genes Dev 16: 2274-2285. doi:10.1101/gad.1007302

Littlepage LE, Wu H, Andresson T, Deanehan JK, Amundadottir LT, Ruderman JV (2002) Identification of phosphorylated residues that affect the activity of the mitotic kinase Aurora-A. Proc Natl Acad Sci U S A 99: 15440-15445. doi:10.1073/pnas.202606599

Mani SA, Guo W, Liao M-J, Eaton Eng, Ayyanan A, Zhou AY, Brooks M, Reinhard F, Zhang CC, Shipitsin M, et al (2008) The epithelial-mesenchymal transition generates cells with properties of stem cells. Cell 133: 704-715. doi:10.1016/j.cell.2008.03.027

Mazumdar A, Henderson YC, El-Naggar AK, Sen S, Clayman GL (2009) Aurora kinase A inhibition and paclitaxel as targeted combination therapy for head and neck squamous cell carcinoma. Head Neck 31: 625-634. doi:10.1002/hed.21007

Melser S, Chatelain EH, Lavie J, MahfoufW, Jose C, Obre E, Goorden S, Priault M, Elgersma Y, Rezvani HR, et al (2013) Rheb regulates mitophagy induced by mitochondrial energetic status. Cell Metab 17: 719-730. doi:10.1016/j.cmet.2013.03.014

Melser S, Lavie J, Bénard G (2015) Mitochondrial degradation and energy metabolism. Biochim Biophys Acta 1853: 2812-2821. doi:10.1016/ j.bbamcr.2015.05.010

Mercer TJ, Gubas A, Tooze SA (2018) A molecular perspective of mammalian autophagosome biogenesis. J Biol Chem 293: 5386-5395. doi:10.1074/ jbc.r117.810366

Mergen M, Engel C, Muller B, Follo M, Schafer T, Jung M, Walz G (2013) The nephronophthisis gene product NPHP2/Inversin interacts with Aurora A and interferes with HDAC6-mediated cilia disassembly. Nephrol Dial Transpl 28: 2744-2753. doi:10.1093/ndt/gft316

Merkwirth C, Dargazanli S, Tatsuta T, Geimer S, Löwer B, Wunderlich FT, von Kleist-Retzow J-C, Waisman A, Westermann B, Langer T (2008) Prohibitins control cell proliferation and apoptosis by regulating OPA1-dependent cristae morphogenesis in mitochondria. Genes Dev 22: 476-488. doi:10.1101/gad.460708

Min M, Mevissen TET, De Luca M, Komander D, Lindon C (2015) Efficient APC/C substrate degradation in cells undergoing mitotic exit depends on K11 ubiquitin linkages. Mol Biol Cell 26: 4325-4332. doi:10.1091/mbc.e15-02-0102

Mishra P, Chan DC (2016) Metabolic regulation of mitochondrial dynamics. J Cell Biol 212: 379-387. doi:10.1083/jcb.201511036

Mlodzik M, Baker NE, Rubin GM (1990) Isolation and expression of scabrous, a gene regulating neurogenesis in Drosophila. Genes Dev 4: 1848-1861. doi:10.1101/gad.4.11.1848

Mortensen M, Ferguson DJP, Edelmann M, Kessler B, Morten KJ, Komatsu M, Simon AK (2010) Loss of autophagy in erythroid cells leads to defective removal of mitochondria and severe anemia in vivo. Proc Natl Acad Sci U S A 107: 832-837. doi:10.1073/pnas.0913170107

Nakatogawa H (2013) Two ubiquitin-like conjugation systems that mediate membrane formation during autophagy. Essays Biochem 55: 39-50. doi:10.1042/bse0550039

Narendra D, Tanaka A, Suen D-F, Youle RJ (2008) Parkin is recruited selectively to impaired mitochondria and promotes their autophagy. I Cell Biol 183: 795-803. doi:10.1083/jcb.200809125

Nikonova AS, Astsaturov I, Serebriiskii IG, Dunbrack RL, Golemis EA (2013) Aurora A kinase (AURKA) in normal and pathological cell division. Cell Mol Life Sci 70: 661-687. doi:10.1007/s00018-012-1073-7 
Novak I, Kirkin V, McEwan DG, Zhang J, Wild P, Rozenknop A, Rogov V, Löhr F, Popovic D, Occhipinti A, et al (2010) Nix is a selective autophagy receptor for mitochondrial clearance. EMBO Rep 11: 45-51. doi:10.1038/embor.2009.256

Padilla-Parra S, Audugé N, Coppey-Moisan M, Tramier M (2008) Quantitative FRET analysis by fast acquisition time domain FLIM at high spatial resolution in living cells. Biophys / 95: 2976-2988. doi:10.1529/ biophysj.108.131276

Paris J, Philippe M (1990) Poly(A) metabolism and polysomal recruitment of maternal mRNAs during early Xenopus development. Dev Biol 140: 221-224. doi:10.1016/0012-1606(90)90070-y

Patrick R, Horin C, Kobe B, Cao K-AL, Bodén M (2016) Prediction of kinasespecific phosphorylation sites through an integrative model of protein context and sequence. Biochim Biophys Acta 1864: 1599-1608. doi:10.1016/j.bbapap.2016.08.001

Pickrell AM, Youle RJ (2015) The roles of PINK1, parkin and mitochondrial fidelity in Parkinson's disease. Neuron 85: 257-273. doi:10.1016/ j.neuron.2014.12.007

Princely Abudu Y, Pankiv S, Mathai BJ, Håkon Lystad A, Bindesb $\varnothing$ ll C, Brenne HB, Yoke Wui Ng M, Thiede B, Yamamoto A, Mutugi Nthiga T, et al (2019) NIPSNAP1 and NIPSNAP2 act as "eat me" signals for mitophagy. Dev Cell 49: 509-525.e12. doi:10.1016/j.devcel.2019.03.013

Pugacheva EN, Golemis EA (2005) The focal adhesion scaffolding protein HEF1 regulates activation of the Aurora-A and Nek2 kinases at the centrosome. Nat Cell Biol 7: 937-946. doi:10.1038/ncb1309

Pugacheva EN, Jablonski SA, Hartman TR, Henske EP, Golemis EA (2007) HEF1Dependent aurora A activation induces disassembly of the primary cilium. Cell 129: 1351-1363. doi:10.1016/j.cell.2007.04.035

Roghi C, Giet R, Uzbekov R, Morin N, Chartrain I, Le Guellec R, Couturier A, Dorée M, Philippe M, Prigent C (1998) The Xenopus protein kinase pEg2 associates with the centrosome in a cell cycle-dependent manner, binds to the spindle microtubules and is involved in bipolar mitotic spindle assembly. J Cell Sci 111: 557-572.

Sasazawa Y, Kanagaki S, Tashiro E, Nogawa T, Muroi M, Kondoh Y, Osada H, Imoto M (2012) Xanthohumol impairs autophagosome maturation through direct inhibition of valosin-containing protein. ACS Chem Biol 7: 892-900. doi:10.1021/cb200492h

Schwarten M, Mohrlüder J, Ma P, Stoldt M, Thielmann Y, Stangler T, Hersch N, Hoffmann B, Merkel R, Willbold D (2009) Nix directly binds to GABARAP: A possible crosstalk between apoptosis and autophagy. Autophagy 5: 690-698. doi:10.4161/auto.5.5.8494

Sehdev V, Katsha A, Ecsedy J, Zaika A, Belkhiri A, El-Rifai W (2013) The combination of alisertib, an investigational Aurora kinase A inhibitor, and docetaxel promotes cell death and reduces tumor growth in preclinical cell models of upper gastrointestinal adenocarcinomas: Alisertib \& docetaxel inhibit tumor growth. Cancer 119: 904-914. doi:10.1002/cncr.27801

Sizaire F, Le Marchand G, Pécréaux J, Bouchareb O, Tramier M (2020) Automated screening of AURKA activity based on a genetically encoded FRET biosensor using fluorescence lifetime imaging microscopy. Methods Appl Fluoresc 8: 024006. doi:10.1088/2050-6120/ab73f5

Soubannier V, McLelland G-L, Zunino R, Braschi E, Rippstein P, Fon EA, McBride HM (2012) A vesicular transport pathway shuttles cargo from mitochondria to lysosomes. Curr Biol 22: 135-141. doi:10.1016/ j.cub.2011.11.057

Tresse E, Salomons FA, Vesa J, Bott LC, Kimonis V, Yao T-P, Dantuma NP, Taylor JP (2010) VCP/p97 is essential for maturation of ubiquitin-containing autophagosomes and this function is impaired by mutations that cause IBMPFD. Autophagy 6: 217-227. doi:10.4161/auto.6.2.11014

Tsunematsu T, Arakaki R, Yamada A, Ishimaru N, Kudo Y (2015) The noncanonical role of aurora-A in DNA replication. Front Oncol 5: 187. doi:10.3389/fonc.2015.00187
Twig G, Shirihai OS (2011) The interplay between mitochondrial dynamics and mitophagy. Antioxid Redox Signal 14: 1939-1951. doi:10.1089/ars.2010.3779

Vaufrey L, Balducci C, Lafont R, Prigent C, Le Bras S (2018) Size matters! Aurora A controls Drosophila larval development. Dev Biol 440: 88-98. doi:10.1016/j.ydbio.2018.05.005

Villa E, Marchetti S, Ricci J-E (2018) No parkin zone: Mitophagy without parkin Trends Cell Biol 28: 882-895. doi:10.1016/j.tcb.2018.07.004

Walter AO, Seghezzi W, Korver W, Sheung J, Lees E (2000) The mitotic serine/ threonine kinase Aurora2/AIK is regulated by phosphorylation and degradation. Oncogene 19: 4906-4916. doi:10.1038/sj.onc.1203847

Wang H, Somers GW, Bashirullah A, Heberlein U, Yu F, Chia W (2006) Aurora-A acts as a tumor suppressor and regulates self-renewal of Drosophila neuroblasts. Genes Dev 20: 3453-3463. doi:10.1101/gad.1487506

Wei Y, Chiang W-C, Sumpter R, Mishra P, Levine B (2017) Prohibitin 2 is an inner mitochondrial membrane mitophagy receptor. Cell 168: 224-238.e10. doi:10.1016/j.cell.2016.11.042

Wirtz-Peitz F, Nishimura T, Knoblich JA (2008) Linking cell cycle to asymmetric division: Aurora-A phosphorylates the Par complex to regulate Numb localization. Cell 135: 161-173. doi:10.1016/j.cell.2008.07.049

Xian H, Yang Q, Xiao L, Shen H-M, Liou Y-C (2019) STX17 dynamically regulated by Fis1 induces mitophagy via hierarchical macroautophagic mechanism. Nat Commun 10: 2059. doi:10.1038/s41467-019-10096-1

Xue Y, Zhou F, Zhu M, Ahmed K, Chen G, Yao X (2005) GPS: A comprehensive www server for phosphorylation sites prediction. Nucleic Acids Res 33 W184-W187. doi:10.1093/nar/gki393

Yan C, Gong L, Chen L, Xu M, Abou-Hamdan H, Tang M, Désaubry L, Song Z (2020) PHB2 (prohibitin 2) promotes PINK1-PRKN/Parkin-dependent mitophagy by the PARL-PGAM5-PINK1 axis. Autophagy 16: 419-434. doi:10.1080/15548627.2019.1628520

Yoshii SR, Kishi C, Ishihara N, Mizushima N (2011) Parkin mediates proteasomedependent protein degradation and rupture of the outer mitochondrial membrane. J Biol Chem 286: 19630-19640. doi:10.1074/jbc.m110.209338

Yoshimaru T, Komatsu M, Tashiro E, Imoto M, Osada H, Miyoshi Y, Honda J, Sasa M, Katagiri T (2015) Xanthohumol suppresses oestrogensignalling in breast cancer through the inhibition of BIG3-PHB2 interactions. Sci Rep 4: 7355. doi:10.1038/srep07355

Youle RJ, Narendra DP (2011) Mechanisms of mitophagy. Nat Rev Mol Cell Biol 12: 9-14. doi:10.1038/nrm3028

Yu X, Minter-Dykhouse K, Malureanu L, Zhao W-M, Zhang D, Merkle CJ, Ward IM, Saya $\mathrm{H}$, Fang G, van Deursen J, et al (2005) Chfr is required for tumor suppression and Aurora A regulation. Nat Genet 37: 401-406. doi:10.1038/ng1538

Zhang J, Randall MS, Loyd MR, Dorsey FC, Kundu M, Cleveland JL, Ney PA (2009) Mitochondrial clearance is regulated by Atg7-dependent and -independent mechanisms during reticulocyte maturation. Blood 114: 157-164. doi:10.1182/blood-2008-04-151639

Zhang Y, Ni J, Huang Q, Ren W, Yu L, Zhao S (2007) Identification of the autoinhibitory domains of Aurora-A kinase. Biochem Biophys Res Commun 357: 347-352. doi:10.1016/j.bbrc.2007.03.129

Zheng F, Yue C, Li G, He B, Cheng W, Wang X, Yan M, Long Z, Qiu W, Yuan Z, et al (2016) Nuclear AURKA acquires kinase-independent transactivating function to enhance breast cancer stem cell phenotype. Nat Commun 7: 10180. doi:10.1038/ncomms10180

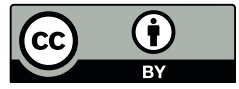

License: This article is available under a Creative Commons License (Attribution 4.0 International, as described at https://creativecommons.org/ licenses/by/4.0/). 